\title{
Development of a PCR based marker system for easy identification and classification of aerobic endospore forming bacilli
}

\author{
Sangeeta Kadyan, Manju Panghal, Khushboo Singh and Jaya Parkash Yadav*
}

\begin{abstract}
Restriction fragment length analysis of 165 rRNA gene of 52 different aerobic endospore forming Bacilli (AEFB) strains with Haelll enzyme has revealed the presence of a 460 bp long fragment in 50 AEFB strains. BLAST analysis revealed that the fragment was 463 bp long and it was located at $3^{\prime}$ end of 165 rRNA gene. Further specificity of this fragment for AEFB strains was checked by PCR and in silico methods. In PCR based method a primer pair (463 F and 463R) specific to this fragment was designed and this primer pair has shown amplification of $463 \mathrm{bp}$ fragment in AEFB strains only. In in silico methods homology of primer pair and presence of restriction enzyme site in 165 rRNA genes were checked in 268 species of AEFB. Almost all species of AEFB have shown positive results for both of the tests. Further multiple alignments of $463 \mathrm{bp}$ sequences of different species of AEFB have shown that it is a good marker for identification and classification of AEFB.
\end{abstract}

Keywords: AEFB; 16 S rRNA gene; Haell; Specificity of fragment; PCR; In silico

\section{Introduction}

Aerobic endospore-formers have long been considered to be important components of the soil bacterial community (Mandic-Mulec and Prosser 2011). There is a great diversity of physiology among the aerobic spore formers. Their collective features include degradation of all substrates derived from plant and animal sources including cellulose, starch, pectin, proteins, agar, hydrocarbons and others, antibiotic production, nitrification, denitrification, nitrogen fixation, facultative lithotrophy, autotrophy, acidophily, alakliphily, psychrophily, thermophily and parasitism. Endospore formation, universally found in this group, is thought to be a strategy for survival even under adverse soil environment, where these bacteria predominate (Kumar et al. 2012). To get the beneficial effects of these AEFB it becomes very necessary to know how much diverse and abundant these microbes are in different soil ecosystems. Since 1990s various approaches based on phenotypic and genotypic characteristics have been applied to identify and classify the members of class Bacilli. Few decades before genus Bacillus was the only representative

\footnotetext{
* Correspondence: yadav1964@rediffmail.com

Postal Address: Department of Genetics, M. D. University, Rohtak 124001, Haryana, India
}

of class Bacilli among aerobic spore formers. Development of cultivation independent approaches have attracted microbiologist towards the molecular approaches for examining the microbes in a better way. Among different molecular methods, $16 \mathrm{~S}$ rRNA gene sequencing is the best one. Since 1991, several new genera of aerobic spore formres like Amphibacillus (Niimura et al. 1990), Paenibacillus (Ash et al. 1991, 1993), Alicyclobacillus (Wisotzkey et al. 1992), Aneurinibacillus (Shida et al. 1996), Brevibacillus (Shida et al. 1996), Gracilibacillus (Waino et al. 1999), Salibacillus (Waino et al. 1999), Virgibacillus (Heyndrickx et al. 1998), Filobacillus (Schlesner et al. 2001), Geobacillus (Nazina et al. 2001), Jeotgalibacillus and Marinibacillus (Yoon et al. 2001) and Ureibacillus (Fortina et al. 2001) have been created based on this method. For phylogenetic arrangement of these newly discovered texa various markers based on 16S rDNA have been developed by different scientists (Priest et al. 1988; Ash et al. 1991; Gurtler and Stanisich 1996; Daffonchio et al. 1998a, b; Goto et al. 2000; Stackebrandt and Swiderski 2002; Xu and Cote 2003; De Clerck et al. 2004; Vardhan et al. 2011). Primer set developed by Garbeva et al. (2003) was found to be $100 \%$ specific for many of species of Bacillus and related genera. After a gap of years, Vardhan et al. (2011)

\section{实 Springer}


developed a set of primers for identification of hyper variable region of $16 \mathrm{~S}$ rDNA in different Bacillus species and partial sequencing of this hyper variable region behaves as an index for easy identification of species related to genera Bacillus.

With development of more advanced approaches to find cultivable and noncultivable diversity of microbes, lot of new species and genera, belonging to AEFB are discovering day by day. So, need of new marker systems is always there for proper identification and classification of these lineages. Hence the main objective of present study was to develop a simple and easy identification and classification tool for Bacillus and related genera which is an extension of research related to bacilli. The restriction digestion of amplified $16 \mathrm{~S}$ rRNA gene by HaeIII enzyme has given a fragment of around $460 \mathrm{bp}$ length in all species of Bacillus and related genera. Sequence information of this fragment (downloaded from $\mathrm{NCBI}$ ) was used to find exact length of the fragment (463 bp) and to develop specific primers for amplification of this fragment in AEFB genera. Further sequence information and multiple alignment of 463 bp long sequences of different species of AEFB genera has revealed that this is an easy tool for identification and classification of the members of Bacillus and related genera. Another beneficial information provided by our study is that almost all species of Bacillus and related genera have restriction enzyme sites for Hae III enzyme which give a product of $460 \mathrm{bp}$. Restriction enzyme site for HaeIII are present at different positions in other bacterial lineages, therefore give product of different size after restriction digestion which clearly discriminate the Bacillus and related genera from others.

\section{Material and methods}

\section{Bacterial strains}

All of the bacterial strains used in the present study are Bacilli isolated from the rhizospheric soil of Phyllanthus amarus which were identified by $16 \mathrm{~S}$ rRNA gene sequencing in our previous research work (Kadyan et al. 2013). Taxonomic information and accession numbers of isolates have been given in Table 1.

\section{S rRNA gene amplification and restriction digestion by Haelll enzyme}

Gene coding for $16 \mathrm{~S}$ rRNA gene of all of the 52 AEFB strains along with 10 reference strains (Shigella Flexneri ATCC12022, Proteus mirabilus ATCC43071, Staphylococcus aureus ATCC259323, E. Coli ATCC25922, Salmonella typhimurium ATCC13311, Klebsiella pneumonia ATCC 700603, Pseudomonas fluorescens MTCC1749, Serretia marcescens MTCC4822, Bacillus subtilis MTCC7193, and Staphylococcus aureus MTCC7443) was amplified by using universal primers i.e. B27f (5'-AGAGTTTGATCCTGGCT
CAG-3') and U1492R (5'- GGTTACCTTGTTACGAC TT-3') in thermal cycler (Biorad). Further reaction mixture for restriction digestion was prepared by mixing $8.5 \mu \mathrm{l}$ of purified PCR products, $5 \mathrm{U}$ of restriction endonuclease, HaeIII (Fermentas) and $1.0 \mu \mathrm{l}$ of $10 \mathrm{X}$ recommendation buffer. Reaction mixture was incubated overnight in water bath at $37^{\circ} \mathrm{C}$. Restriction digested DNA was analysed by horizontal electrophoresis in 2\% agarose gels with 100 bp DNA marker. The gels were visualized on a gel documentation system (Alpha Innotech). Photograph of gel has been shown in Figure 1(a\&b).

\section{Restriction pattern analysis and designing of oligonucleotide primers}

Restriction pattern analysis of HaeIII digested $16 \mathrm{~S}$ rRNA gene has shown the presence of a fragment having length around $460 \mathrm{bp}$ (Figure 1a) in all of the bacterial species belonging to Bacillus and related genera (except Bacillus arsenicus, Paenibacillus taiwanensis and 9 reference strains related to other bacterial lineages) (Figure 1b). On the basis of these observations it was assumed that this $460 \mathrm{bp}$ fragment was specific for Bacillus and related genera. To find out the exact location and sequence information of this fragment, $16 \mathrm{~S}$ rRNA gene sequence of all of the Bacillus isolates taken in our study was downloaded from NCBI gene bank database. All of the $16 \mathrm{~S}$ rRNA gene sequences were checked for HaeIII enzyme cut sites (GG $\downarrow C C)$. Sequence between two cut sites having length of around $460 \mathrm{bp}$ was found in all of the $16 \mathrm{~S}$ rRNA gene sequences at same position. Further length of this region was found to be 461-463 bp. Primer pair specific to this region was designed by using software, Primer 3.0 and further synthesized from the facility available at Eurofins Genomics India Pvt. Ltd., Bangalore.

\section{Sequence specificity of primer pair and occurrence of restriction enzyme site}

The specificity of oligonucleotide primers was checked by PCR amplification of the $463 \mathrm{bp}$ fragment in all of the 52 AEFB isolates along with 10 reference strains (Shigella Flexneri ATCC12022, Proteus mirabilus ATCC43071, Staphylococcus aureus ATCC259323, E. Coli ATCC25922, Salmonella typhimurium ATCC13311, Klebsiella pneumonia ATCC 700603, Pseudomonas fluorescens MTCC1749, Serretia marcescens MTCC4822, Bacillus subtilis MTCC7193, and Staphylococcus aureus MTCC7443). Reaction conditions for PCR were, initial denaturation at $94^{\circ} \mathrm{C}$ for 5 minutes, 30 cycles of denaturation at $95^{\circ} \mathrm{C}$ for 30 seconds, annealing at $55^{\circ} \mathrm{C}$ for 20 seconds, extension at $72^{\circ} \mathrm{C}$ for 30 seconds and at last final extension at $72^{\circ} \mathrm{C}$ for 7 minutes. Theoretically primer pair was checked for its specificity in 16S rRNA gene sequences (downloaded from NCBI) in different species of Bacillus and related genera i.e. 153 different species of Bacillus, 20 Virgibacillus, 15 
Table 1 Strain names and NCBI accession numbers of 52 AEFB strains isolated from rhizospheric soil of Phyllanthus amarus

\begin{tabular}{|c|c|c|c|c|c|}
\hline Strain code & Bacterial isolate & Accession number & Strain code & Bacterial isolate & Accession number \\
\hline 1.P3 & B. marisflavi JP44SK40 & JX129227 & $15 . P 2$ & B. subtilis subsp. spizizenii JP44SK24 & $J X 144714$ \\
\hline 2.P1 & B. megaterium JP44SK1 & $J X 144691$ & 16.P1 & B. simplex JP44SK25 & $J X 144715$ \\
\hline 2.P2 & B. megaterium JP44SK2 & $J X 144692$ & $16 . P 2$ & B. simplex JP44SK26 & JX144716 \\
\hline 3.P1 & Lysinibacillus sphaericus JP44SK3 & $J X 144693$ & 17.P3 & B. cereus JP44SK27 & $J X 144717$ \\
\hline 3.P2 & Lysinibacillus sphaericus JP44SK4 & $J X 144694$ & 18.P3 & B. aquimaris JP44SK28 & $J X 144718$ \\
\hline 3.P3 & B. megaterium JP44SK5 & $J X 144695$ & 19.P1 & B. simplex JP44SK29 & $J X 144719$ \\
\hline 4.P1 & B. licheniformis JP44SK6 & $J X 144696$ & 19.P2 & B. simplex JP44SK30 & $J X 144720$ \\
\hline 5.P3 & Paenibacillus taiwanensis JP44SK7 & JX144697 & 20.P1 & B. simplex JP44SK31 & $J X 144721$ \\
\hline 6.P1 & B. mycoides JP44SK8 & JX144698 & $20 . P 2$ & B. simplex JP44SK32 & JX144722 \\
\hline 6.P3 & B. mycoides JP44SK9 & $J X 144699$ & 23.P1 & B. cereus JP44SK33 & $J X 144723$ \\
\hline 7.P1 & B. aryabhattai JP44SK11 & $J X 144701$ & 23.P2 & B. cereus JP44SK34 & $J X 144724$ \\
\hline 7.P2 & B. megaterium JP44SK10 & $J X 144700$ & 23.P3 & B. megaterium JP44SK35 & $J X 144725$ \\
\hline 7.P3 & Lysinibacillus xylanilyticus JP44SK52 & $J X 155769$ & 24.P1 & B. mycoides JP44SK36 & $J X 144726$ \\
\hline 8.P1 & B. simplex JP44SK12 & $J X 144702$ & 24.P3 & B. cereus JP44SK37 & $J X 144727$ \\
\hline 8.P2 & B. simplex JP44SK13 & $J \times 144703$ & 25.P2 & B. aryabhattai JP44SK38 & $J X 144728$ \\
\hline 8.P3 & B. arsenicus JP44SK14 & $J X 144704$ & $26 . P 3$ & B. megaterium JP44SK39 & $J X 144729$ \\
\hline $9 . \mathrm{P3}$ & B. marisflavi JP44SK15 & $J X 144705$ & 27.P1 & Brevibacillus laterosporus JP44SK41 & $J X 155758$ \\
\hline 10.P3 & B. firmus JP44SK16 & JX144706 & 27.P3 & B. cereus JP44SK42 & $J X 155759$ \\
\hline 11.P1 & B. firmus JP44SK17 & $J X 144707$ & 30.P1 & B. cereus JP44SK43 & $J X 155760$ \\
\hline 11.P3 & B. megaterium JP44SK18 & $J X 144708$ & 31.P3 & Jeotgalibacillus sp. JP44SK56 & KC012993 \\
\hline 12.P3 & B. flexus JP44SK19 & $J X 144709$ & $36 . P 3$ & B. cereus JP44SK44 & $J \times 155761$ \\
\hline 13.P1 & B. megaterium strain JP44SK21 & $J X 144711$ & 37.P3 & B. cereus JP44SK45 & $J X 155762$ \\
\hline 13.P3 & B. firmus JP44SK20 & JX144710 & 38.P3 & Terribacillus saccharophilus JP44SK46 & JX155763 \\
\hline 14.P2 & Brevibacillus laterosporus JP44SK51 & $J X 155768$ & 41.P3 & Terribacillus goriensis JP44SK47 & $J X 155764$ \\
\hline 14.P3 & B. cereus JP44SK22 & $J X 144712$ & 43.P3 & B. cereus JP44SK49 & $J X 155766$ \\
\hline 15.P1 & B. subtilis subsp. spizizenii JP44SK23 & $J \times 144713$ & $44 . \mathrm{P3}$ & B. mycoides JP44SK50 & JX155767 \\
\hline
\end{tabular}

Geobacillus, 1 Filobacillus, 4 Jeotgalibacillus, 5 Ureibacillus, 21 Alicyclobacillus, 5 Amphibacillus, 5 Aneurinibacillus, 16 Brevibacillus, 9 Gracilibacillus, 5 Paenibacillus, 5 Lysinibacillus and 4 Terribacillus. A number of other bacterial lineages of Gram positive and negative bacteria were also checked for primer specificity which includes genera from phylum Firmicutes (other than Bacilli), Actinobacteria, Alpha Proteobacteria, Beta Proteobacteria and Gamma Proteobacteria.

\section{Multiple alignment of 463 bp long partial 16S rDNA sequence of different species of Bacilli}

To check the ability of marker for classification of Bacilli, we have done the multiple alignments of specific, $463 \mathrm{bp}$ long sequences of $16 \mathrm{~S}$ rRNA gene of 52 strains (taken in our study) with the reference sequences downloaded from NCBI. Multiple alignment of very closely related species of genus Bacillus (29 different species of Bacillus) lying in two nearby clusters in all species living tree by Yarza et al.
(2010) has also been done to check the differentiation ability of this sequence. Software Clustal X 2.0 (Larkin et al. 2007) was used for alignment of different sequences and further alignment file was used in molecular evolutionary genetic analysis software version 5.1 (MEGA 5.1) (Tamura et al. 2011) for construction of phylogenetic tree.

\section{Results}

\section{Oligonucleotide primers}

Bacillus and related genera specific primers designed in our study were named as $463 \mathrm{~F}$ (5'CTAAAACTCAAAG GAATTGACG3') and 463R (5'AATACGTTCCCGG GCCTT3').

\section{PCR amplification of 463 bp sequence}

PCR amplification has confirmed the specificity of the primer pair in 52 AEFB strains and 10 reference strains. Out of total, 50 strains belonging to Bacillus and related genera have shown the amplification of the specific 


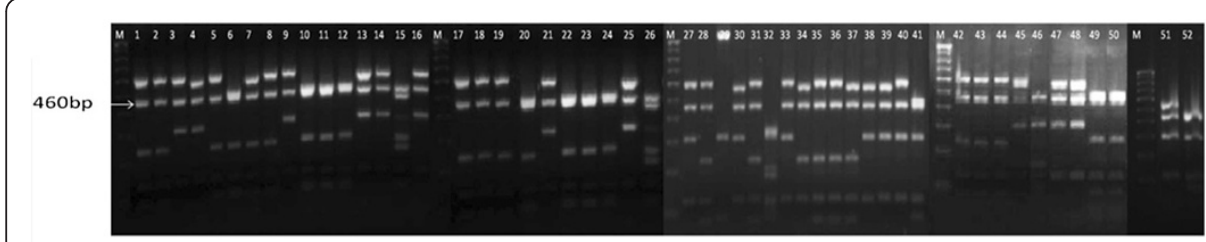

(a)

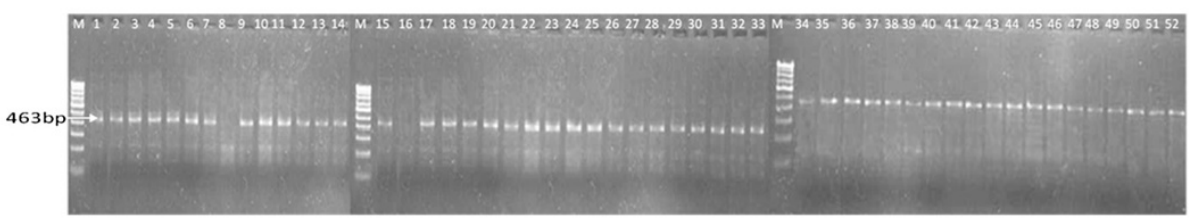

(c)

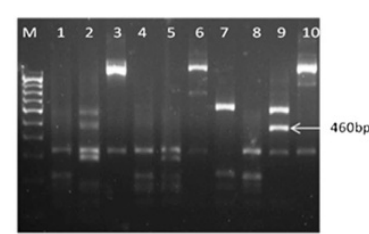

(b)

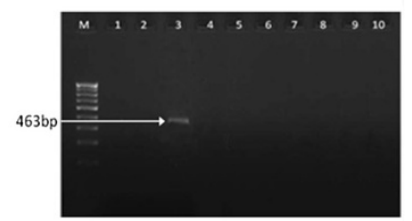

(d)

Figure 1 Gel photograph showing ARDRA pattern of 52 AEFB strains (a) Gel photograph of ARDRA pattern of 52 AEFB strains digested with Hae III restriction enzyme. (b) Gel photograph of ARDRA pattern of 10 reference strains digested with Hae III restriction enzyme. (c) Gel photograph of PCR amplified 463 bp fragments in 52 strains of AEFB. (d) Gel photograph of PCR amplification result of 463 bp fragments in 10 reference strains. (a) Lane M - 100 bp DNA marker. Lanes 1-52 indicate bacterial strain codes (2.P1, 3.P1, 4.P1, 6.P1, 7.P1, 8.P1, 11.P1, 13.P1, 15.P1, 16.P1, 19.P1, 20.P1, 23.P1, 24.P1, 27.P1, 30.P1, M, 2.P2, 3.P2, 7.P2, 8.P2, 15.P2, 16.P2, 19.P2, 20.P2, 23.P2, 14.P2, M, 1.P3, 3.P3, 5.P3, 6.P3, 7.P3, 8.P3, 9.P3, 10.P3, 11.P3, 12.P3, 13.P3, 14.P3, 17.P3, 18.P3, 24.P3, M, 23.P3, 25.p2, 26.P3, 27.P3, 31.P3, 36.P3, 37.P3, 38.P3, 41.P3, 43.p3, 44.p3). (b) Lane M -100 bp DNA marker, lane 1-10 Shigella flexneri ATCC12022, Proteus mirabilus ATCC43071, Staphylococcus aureus ATCC259323, E. Coli ATCC25922,

Salmonella typhimurium ATCC13311, Klebsiella pneumoniae ATCC 700603, Pseudomonas fluorescens MTCC1749, Serretia marrcescens MTCC4822, Bacillus subtilis MTCC7193, Staphylococcus aureus MTCC7443. Arrow indicates the size of 460 bp fragment in Bacillus subtilis MTCC7193. (c): M 100 bp DNA marker, lane 1-52 (1.P3, 2.P1, 2.P2, 3.P1, 3.P2, 3.P3, 4.P1, 5.P3, 6.P1, 6.P3, 7.P1, 7.P2, 7.P3, 8.P1, 8.P2, 8.P3, 9.P3, 10.P3, 11.P1, 11.P3, 12.P3, 13.P1, 13.P3, 14.P2, 14.P3, 15.P1, 15.P2, 16.P1, 16.P2, 17.P3, 18.P3, 19.P1, 19.P2, 20.P1, 20.P2, 23.P1, 23.P2, 23.P3, 24.P1, 24.P3, 25.P2, 26.P3, 27.P1, 27.P3, 30.P1, 31.P3, 36.P3, 37.P3, 38.P3, 41.P3, 43.P3, 44.P3). Arrow indicates the size of fragment. (d): Bacterial strain Bacillus subtilis MTCC7193, present in lane no. 3 has shown amplification of $463 \mathrm{bp}$ fragment and other reference strains have not shown any amplification. Arrow indicates the size of fragment compared with marker of 100 bp present in lane M.

region. However, the region was not amplified in Bacillus arsenicus, Paenibacillus taiwanensis and 9 reference strains (Figure 1c \& d).

\section{Sequence homology of primers in 16S rRNA gene sequences of Bacillus and related genera}

Primer sequences were found to be $100 \%$ similar with the 16S rRNA gene sequences (downloaded from NCBI) of 120 species of genera Bacillus, 13 Geobacillus, 1 Filobacillus, 4 Jeotgalibacillus, 5 Ureibacillus, 7 Alicyclobacillus, 2 Brevibacillus and 5 Lysinibacillus. Number of other bacterial lineages of Gram positive and negative bacteria which includes genera from phyla Firmicutes (Staphylococcus chromogenes D83360, Streptococcus pyogenes AB002521, Enterococcus faecalis AB012212, Clostridium populeti X71853, Listeria monocytogenes X56153), Actinobacteria (Corynebacterium diphtheria X84248, Mycobacterium tuberculosis X58890, Nocardia asteroids AF430019, Streptomyces lavendulae subsp. Lavendulae D85116), Alpha proteobacteria (Rhizobium leguminosarum U29386, Azospirillum lipoferum Z29619, Acetobacterium woodii X96954), Beta proteobacteria (Burkholderia cepacia U96927, Bordetella pertussis U04950) and Gamma Proteobacteria (Pseudomonas aeruginosa X06684, Escherichia coli X80725, Klebsiella pneumoniae X87276, Shigella dysenteriae X96966) have not shown any sequence homology (Table 2).

\section{Multiple alignments of $463 \mathrm{bp}$ sequences of different strains of Bacilli}

Dendrogram prepared on the basis of alignment of $463 \mathrm{bp}$ sequence has been given in Figure 2(a\&b). Dendrogram prepared for 52 different strains of Bacillus and related genera (taken in our study) and some reference sequences downloaded from NCBI has been shown in Figure 2(a). Dendrogram has been divided in to 7 different groups (I-VII). Group I contains strains belonging to species Bacillus aquimaris and marisflavi. Strains belonging to genera Lysinibacillus (sphaericus and xylanilyticus) and Jeotgalibacillus are present in Group II. Group III contains strains belonging to Genera Terribacillus (sacharrophilus and goriensis), Bacillus subtilis sub sp. spizizinii and Bacillus licheniformis. Group IV contains strains belonging to species, Bacillus mycoides and Bacillus cereus. Group V contains strains belonging to genera Paenibacillus and Brevibacillus and strains belonging to species Bacillus simplex and Bacillus firmus have shared the group VI. Bacillus arsenicus has not shown any grouping with any other species or genera and Bacillus megaterium and Bacillus flexus have shared a single group VII while some strains of Bacillus megaterium, Bacillus flexus and Bacillus 
Table $2 \%$ similarity of 463 bp sequence of 16S rRNA gene of type sp. (Bacillus subtilis) with 16S rRNA sequences of different AEFB strains (downloaded from NCBI), primer sequences in these AEFB strains, presence and absence of restriction enzyme site and position of specific fragment in AEFB strains

\begin{tabular}{|c|c|c|c|c|c|}
\hline Sr. no. & Name of bacteria & NCBI accession no. & $\begin{array}{l}\text { Sequence of primer pair } \\
\text { in different } A E F B \text { strains }\end{array}$ & $\begin{array}{l}\% \text { similarity of } 463 \mathrm{bp} \\
\text { sequence and presence } \\
\text { of restriction enzyme site }\end{array}$ & $\begin{array}{l}\text { Position of } 463 \mathrm{bp} \\
\text { sequence in } 16 \mathrm{~S} \\
\text { rRNA gene }\end{array}$ \\
\hline 1 & Alicyclobacillus sacchari & AB264020 & $\begin{array}{l}\text { AATCCGTTCCCGGGCCTT } \\
\text { CTGAAACTCAAAGGAATTGACG }\end{array}$ & $88 \%+$ & $913-1374$ \\
\hline 2 & Alicyclobacillus acidiphilus & AB076660 & $\begin{array}{l}\text { AATCCGTTCCCGGGCCTT } \\
\text { CTGAAACTCAAAGGAATTGACG }\end{array}$ & $88 \%-$ & $913-1374$ \\
\hline 3 & Alicyclobacillus acidoterrestris & AB042057 & $\begin{array}{l}\text { AATCCGTTCCCGGGCCTT } \\
\text { CTGAAACTCAAAGGAATTGACG }\end{array}$ & $88 \%+$ & 910-1371 \\
\hline 4 & Alicyclobacillus hesperidum & AJ133633 & $\begin{array}{l}\text { AATCCGTTCCCGGGCCTT } \\
\text { CTGAAACTCAAAGGAATTGACG }\end{array}$ & $88 \%+$ & 884-1345 \\
\hline 5 & Alicyclobacillus fastidiosus & AB264021 & $\begin{array}{l}\text { AATCCGTTCCCGGGCCTT } \\
\text { CTGAAACTCAAAGGAATTGACG }\end{array}$ & $88 \%+$ & 910-1371 \\
\hline 6 & Alicyclobacillus vulcanalis & AY425985 & $\begin{array}{l}\text { AATACGTTCCCGGGCCTT } \\
\text { CTGAAACTCAAAGGAATTGACG }\end{array}$ & $88 \%+$ & 894-1355 \\
\hline 7 & Alicyclobacillus sendaiensis & AB084128 & $\begin{array}{l}\text { AATACGTTCCCGGGCCTT } \\
\text { CTGAAACTCAAAGGAATTGACG }\end{array}$ & $88 \%+$ & $885-1346$ \\
\hline 8 & Alicyclobacillus contaminans & AB264026 & $\begin{array}{l}\text { AATCCGTTCCCGGGCCTT } \\
\text { CTGAAACTCAAAGGAATTGACG }\end{array}$ & $88 \%+$ & $925-1386$ \\
\hline 9 & $\begin{array}{l}\text { Alicyclobacillus acidocaldarius } \\
\text { subsp. acidocaldarius (Type sp) }\end{array}$ & AJ496806 & $\begin{array}{l}\text { AATACGTTCCCGGGCCTT } \\
\text { CTGAAACTCAAAGGAATTGACG }\end{array}$ & $87 \%+$ & $902-1363$ \\
\hline 10 & Alicyclobacillus aeris & FM179383 & $\begin{array}{l}\text { AATCCGTTCCCGGGCCTT } \\
\text { CTGAAACTCAAAGGAATTGACG }\end{array}$ & $88 \%+$ & $911-1372$ \\
\hline 11 & Alicyclobacillus pomorum & AB089840 & $\begin{array}{l}\text { AATCCGTTCCCGGGCCTT } \\
\text { CTGAAACTCAAAGGAATTGACG }\end{array}$ & $88 \%+$ & $911-1372$ \\
\hline 12 & Alicyclobacillus disulfidooxidans & AB089843 & $\begin{array}{l}\text { AATCCGTTCCCGGGCCTT } \\
\text { CTGAAACTCAAAGGAATTGACG }\end{array}$ & $85 \%+$ & $911-1372$ \\
\hline 13 & Alicyclobacillus tolerans & Z21979 & $\begin{array}{l}\text { AATACGTTCCCGGGCCTT } \\
\text { CTGAAACTCAAAGGAATTGACG }\end{array}$ & $87 \%-$ & $906-1365$ \\
\hline 14 & Alicyclobacillus ferrooxydans & EU137838 & $\begin{array}{l}\text { AATCCGTTCCCGGGCCTT } \\
\text { CTGAAACTCAAAGGAATTGACG }\end{array}$ & $88 \%+$ & $913-1374$ \\
\hline 15 & Alicyclobacillus cycloheptanicus & AB042059 & $\begin{array}{l}\text { AATCCGTTCCCGGGCCTT } \\
\text { CTGAAACTCAAAGGAATTGACG }\end{array}$ & $88 \%+$ & $911-1372$ \\
\hline 16 & Alicyclobacillus macrosporangiidus & AB264025 & $\begin{array}{l}\text { AATACGTTCCCGGGCCTT } \\
\text { CTGAAACTCAAAGGAATTGACG }\end{array}$ & $87 \%-$ & $927-1388$ \\
\hline 17 & Alicyclobacillus kakegawensis & AB264022 & $\begin{array}{l}\text { AATCCGTTCCCGGGCCTT } \\
\text { CTGAAACTCAAAGGAATTGACG }\end{array}$ & $86 \%+$ & $924-1385$ \\
\hline 18 & Alicyclobacillus shizuokensis & AB264024 & $\begin{array}{l}\text { AATCCGTTCCCGGGCCTT } \\
\text { CTGAAACTCAAAGGAATTGACG }\end{array}$ & $87 \%+$ & $924-1385$ \\
\hline 19 & Alicyclobacillus herbarius & AB042055 & $\begin{array}{l}\text { AATCCGTTCCCGGGCCTT } \\
\text { CTGAAACTCAAAGGAATTGACG }\end{array}$ & $87 \%+$ & $924-1385$ \\
\hline 20 & Alicyclobacillus pohliae & AJ564766 & $\begin{array}{l}\text { AATACGTTCCCGGGCCTT } \\
\text { CTGAAACTCAAAGGAATTGACG }\end{array}$ & $88 \%+$ & $904-1363$ \\
\hline 21 & Alicyclobacillus tolerans & Z21979 & $\begin{array}{l}\text { AATACGTTCCCGGGCCTT } \\
\text { CTGAAACTCAAAGGAATTGACG }\end{array}$ & $87 \%-$ & $906-1365$ \\
\hline 22 & Amphibacillus sediminis & AB243866 & $\begin{array}{l}\text { AATACGTTCCCGGGTCTT } \\
\text { CTGAAACTCAAAAGAATTGACG }\end{array}$ & $96 \%-$ & $928-1386$ \\
\hline 23 & Amphibacillus jilinensis, & FJ169626 & $\begin{array}{l}\text { AATACGTTCCCGGGTCTT } \\
\text { CTGAAACTCAAAAGAATTGACG }\end{array}$ & $95 \%-$ & $948-1406$ \\
\hline 24 & Amphibacillus tropicus & AF418602 & $\begin{array}{l}\text { AATACGTTCCCGGGTCTT } \\
\text { CTGAAACTCAAAAGAATTGACG }\end{array}$ & $95 \%-$ & $905-1362$ \\
\hline 25 & Amphibacillus fermentum & AF418603 & $\begin{array}{l}\text { CTGAAACTCAAAGGAATTGACG } \\
\text { AATACGTTCCCGGGTCTT }\end{array}$ & $93 \%-$ & 910-1368 \\
\hline
\end{tabular}


Table $2 \%$ similarity of 463 bp sequence of 16S rRNA gene of type sp. (Bacillus subtilis) with 16S rRNA sequences of different AEFB strains (downloaded from NCBI), primer sequences in these AEFB strains, presence and absence of restriction enzyme site and position of specific fragment in AEFB strains (Continued)

\begin{tabular}{|c|c|c|c|c|c|}
\hline 26 & Amphibacillus xylanus, type sp. & D82065 & $\begin{array}{l}\text { AATACGTTCCCGGGTCTT } \\
\text { CTGAAACTCAAAAGAATTGACG }\end{array}$ & $94 \%-$ & $948-1406$ \\
\hline 27 & $\begin{array}{l}\text { Aneurinibacillus aneurinilyticus } \\
\text { type sp. }\end{array}$ & X94194 & $\begin{array}{l}\text { AATACGTTCCCGGGTCTT } \\
\text { CTGAAACTCAAAGGAATTGACG }\end{array}$ & 91\%- & $903-1369$ \\
\hline 28 & Aneurinibacillus migulanus & X94195 & $\begin{array}{l}\text { CTGAAACTCAAAGGAATTGACG } \\
\text { AATACGTTCCCGGGTCTT }\end{array}$ & $90 \%-$ & $903-1359$ \\
\hline 29 & Aneurinibacillus danicus & $A B 112725$ & $\begin{array}{l}\text { CTGAAACTCAAAGGAATTGACG } \\
\text { AATACGTTCCCGGGTCTT }\end{array}$ & $91 \%-$ & $903-1354$ \\
\hline 30 & Aneurinibacillus thermoaerophilus & X94196 & $\begin{array}{l}\text { CTGAAACTCAAAGGAATTGACG } \\
\text { AATACGTTCCCGGGTCTT }\end{array}$ & $92 \%-$ & 904- 1361 \\
\hline 31 & Aneurinibacillus terranovensis & AJ715385 & $\begin{array}{l}\text { CTGAAACTCAAAGGAATTGACG } \\
\text { AATACGTTCCCGGGTCTT }\end{array}$ & $91 \%-$ & $897-1353$ \\
\hline 32 & Brevibacillus centrosporus & D78458 & $\begin{array}{l}\text { GTGAAACTCAAAGGAATTGACG } \\
\text { AATACGTTCCCGGGCCTT }\end{array}$ & $91 \%+$ & $917-1377$ \\
\hline 33 & Brevibacillus choshinensis & $A B 112713$ & $\begin{array}{l}\text { GTGAAACTCAAAGGAATTGACG } \\
\text { AATACGTTCCCGGGCCTT }\end{array}$ & $91 \%+$ & 894-1354 \\
\hline 34 & Brevibacillus reuszeri & AB112715 & $\begin{array}{l}\text { GTGAAACTCAAAGGAATTGACG } \\
\text { AATACGTTCCCGGGCCTT }\end{array}$ & $91 \%+$ & 894-1354 \\
\hline 35 & Brevibacillus parabrevis & $A B 112714$ & $\begin{array}{l}\text { GTGAAACTCAAAGGAATTGACG } \\
\text { AATACGTTCCCGGGCCTT }\end{array}$ & $91 \%+$ & 894-1354 \\
\hline 36 & Brevibacillus brevis type sp. & AB271756 & $\begin{array}{l}\text { GTGAAACTCAAAGGAATTGACG } \\
\text { AATACGTTCCCGGGCCTT }\end{array}$ & $91 \%+$ & 896-1356 \\
\hline 37 & Brevibacillus formosus & $A B 112712$ & $\begin{array}{l}\text { GTGAAACTCAAAGGAATTGACG } \\
\text { AATACGTTCCCGGGCCTT }\end{array}$ & $91 \%+$ & 894-1354 \\
\hline 38 & Brevibacillus agri & $A B 112716$ & $\begin{array}{l}\text { GTGAAACTCAAAGGAATTGACG } \\
\text { AATACGTTCCCGGGCCTT }\end{array}$ & $91 \%+$ & 895-1355 \\
\hline 39 & Brevibacillus limnophilus & $A B 112717$ & $\begin{array}{l}\text { GTGAAACTCAAAGGAATTGACG } \\
\text { AATACGTTCCCGGGCCTT }\end{array}$ & $91 \%+$ & $909-1369$ \\
\hline 40 & Brevibacillus invocatus & AF378232 & $\begin{array}{l}\text { CTGAAACTCAAAGGAATTGACG } \\
\text { AATACGTTCCCGGGCCTT }\end{array}$ & $91 \%+$ & 896-1356 \\
\hline 41 & Brevibacillus panacihumi & EU383033 & $\begin{array}{l}\text { CTGAAACTCAAAGGAATTGACG } \\
\text { AATACGTTCCCGGGCCTT }\end{array}$ & $91 \%+$ & $902-1362$ \\
\hline 42 & Brevibacillus borstelensis & AB112721 & $\begin{array}{l}\text { GTGAAACTCAAAGGAATTGACG } \\
\text { AATACGTTCCCGGGCCTT }\end{array}$ & $92 \%+$ & 894-1354 \\
\hline 43 & Brevibacillus ginsengisoli & AB245376 & $\begin{array}{l}\text { GTGAAACTCAAAGGAATTGACG } \\
\text { AATACGTTCCCGGGCCTT }\end{array}$ & $92 \%+$ & 873-1333 \\
\hline 44 & Brevibacillus laterosporus & D16271 & $\begin{array}{l}\text { GTGAAACTCAAAGGAATTGACG } \\
\text { AATACGTTCCCGGGCCTT }\end{array}$ & $91 \%+$ & 896-1356 \\
\hline 45 & Brevibacillus fluminis & EU375457 & $\begin{array}{l}\text { GTGAAACTCAAAGGAATTGACG } \\
\text { AATACGTTCCCGGGCCTT }\end{array}$ & $91 \%+$ & 896-1356 \\
\hline 46 & Brevibacillus levickii & AJ715378 & $\begin{array}{l}\text { GTGAAACTCAAAGGAATTGACG } \\
\text { AATACGTTCCCGGGCCTT }\end{array}$ & $91 \%+$ & $897-1357$ \\
\hline 47 & Brevibacillus thermoruber & Z26921 & $\begin{array}{l}\text { GTGAAACTCAAAGGAATTGACG } \\
\text { AATACGTTCCCGGGCCTT }\end{array}$ & $92 \%+$ & $915-1376$ \\
\hline 48 & Gracilibacillus lacisalsi & DQ664540 & $\begin{array}{l}\text { CTGAAACTCAAAAGAATTGACG } \\
\text { AATACGTTCCCGGGCCTT }\end{array}$ & $94 \%+$ & 933-1393 \\
\hline 49 & Gracilibacillus thailandensis & FJ182214 & $\begin{array}{l}\text { CTGAAACTCAAAAGAATTGACG } \\
\text { AATACGTTCCCGGGCCTT }\end{array}$ & $94 \%+$ & $942-1402$ \\
\hline 50 & Gracilibacillus saliphilus & EU784646 & $\begin{array}{l}\text { CTGAAACTCAAAAGAATTGACG } \\
\text { AATACGTTCCCGGGCCTT }\end{array}$ & $94 \%+$ & $917-1377$ \\
\hline 51 & Gracilibacillus orientalis & AM040716 & $\begin{array}{l}\text { CTGAAACTCAAAAGAATTGACG } \\
\text { AATACGTTCCCGGGCCTT }\end{array}$ & $93 \%+$ & $931-1391$ \\
\hline
\end{tabular}


Table $2 \%$ similarity of 463 bp sequence of 16S rRNA gene of type sp. (Bacillus subtilis) with 16S rRNA sequences of different AEFB strains (downloaded from NCBI), primer sequences in these AEFB strains, presence and absence of restriction enzyme site and position of specific fragment in AEFB strains (Continued)

\begin{tabular}{|c|c|c|c|c|c|}
\hline 52 & Gracilibacillus dipsosauri & AB101591 & $\begin{array}{l}\text { CTGAAACTCAAAAGAATTGACG } \\
\text { AATACGTTCCCGGGCCTT }\end{array}$ & $95 \%+$ & $923-1383$ \\
\hline 53 & Gracilibacillus ureilyticus & EU709020 & $\begin{array}{l}\text { CTGAAACTCAAAAGAATTGACG } \\
\text { AATACGTTCCCGGGCCTT }\end{array}$ & $95 \%+$ & $923-1383$ \\
\hline 54 & Gracilibacillus boraciitolerans & AB197126 & $\begin{array}{l}\text { CTGAAACTCAAAAGAATTGACG } \\
\text { AATACGTTCCCGGGCCTT }\end{array}$ & $94 \%+$ & $935-1395$ \\
\hline 55 & $\begin{array}{l}\text { Gracilibacillus halotolerans type } \\
\text { sp. }\end{array}$ & AF036922 & $\begin{array}{l}\text { CTGAAACTCAAAAGAATTGACG } \\
\text { AATACGTTCCCGGGCCTT }\end{array}$ & $94 \%+$ & 934-1394 \\
\hline 56 & Gracilibacillus halophilus & EU135704 & $\begin{array}{l}\text { CTGAAACTCAAAAGAATTGACG } \\
\text { AATACGTTCCCGGGCCTT }\end{array}$ & $94 \%+$ & $924-1384$ \\
\hline 57 & Paenibacillus polymyxa type sp. & D16276 & $\begin{array}{l}\text { AATACGTTCCCGGGTCTT } \\
\text { CTGAAACTCAAAGGAATTGACG }\end{array}$ & $90 \%-$ & $913-1375$ \\
\hline 58 & Paenibacillus antarcticus & AJ605292 & $\begin{array}{l}\text { AATACGTTCCCGGGTCTT } \\
\text { CTGAAACTCAAAGGAATTGACG }\end{array}$ & $90 \%-$ & $915-1374$ \\
\hline 59 & $\begin{array}{l}\text { Paenibacillus macquariensis } \\
\text { subsp. macquariensis }\end{array}$ & X60625 & $\begin{array}{l}\text { AATACGTTCCCGGGTCTT } \\
\text { CTGAAACTCAAAGGAATTGACG }\end{array}$ & $90 \%-$ & $935-1394$ \\
\hline 60 & $\begin{array}{l}\text { Paenibacillus macquariensis } \\
\text { subsp. defensor }\end{array}$ & AB360546 & $\begin{array}{l}\text { AATACGTTCCCGGGTCTT } \\
\text { CTGAAACTCAAAGGAATTGACG }\end{array}$ & $90 \%+$ & 936-1395 \\
\hline 61 & Paenibacillus glacialis & EU815294 & $\begin{array}{l}\text { AATACGTTCCCGGGTCTT } \\
\text { CTGAAACTCAAAGGAATTGACG }\end{array}$ & 91\%- & 934-1393 \\
\hline 62 & $\begin{array}{l}\text { Virgibacillus pantothenticus type } \\
\text { sp. }\end{array}$ & D16275 & $\begin{array}{l}\text { AATACGTTCCCGGGTCTT } \\
\text { CTGAAACTCAAAGGAATTGACG }\end{array}$ & 95\%- & $919-1375$ \\
\hline 63 & Virgibacillus proomii & AJ012667 & $\begin{array}{l}\text { CTGAAACTCAAAAGAATTGACG } \\
\text { AATACGTTCCCGGGTCTT }\end{array}$ & 95\%- & $916-1372$ \\
\hline 64 & Virgibacillus salexigens & Y11603 & $\begin{array}{l}\text { CTGAAACTCAAAAGAATTNACG } \\
\text { AATACGTTCCCGGGCCTT }\end{array}$ & $95 \%+$ & $921-1379$ \\
\hline 65 & Virgibacillus marismortui & AJ009793 & $\begin{array}{l}\text { AATACGTTCCCGGGCCT } \\
\text { CTGAAACTCAAAAGAATTGACG }\end{array}$ & $95 \%+$ & $947-1407$ \\
\hline 66 & Virgibacillus salarius & AB197851 & $\begin{array}{l}\text { AATACGTTCCCGGGCCT } \\
\text { CTGAAACTCAAAAGAATTGACG }\end{array}$ & $95 \%+$ & $949-1409$ \\
\hline 67 & Virgibacillus olivae & DQ139839 & $\begin{array}{l}\text { AATACGTTCCCGGGCCTT } \\
\text { CTGAAACTCAAAAGAATTGACG }\end{array}$ & $95 \%+$ & $948-1409$ \\
\hline 68 & Virgibacillus halodenitrificans & AY543169, & $\begin{array}{l}\text { CTGAAACTCAAAAGAATTGACG } \\
\text { AATACGTTCCCGGGCCTT }\end{array}$ & $95 \%+$ & $926-1386$ \\
\hline 69 & Virgibacillus koreensis & AY616012 & $\begin{array}{l}\text { CTGAAACTCAAAAGAATTGACG } \\
\text { AATACGTTCCCGGGCCTT }\end{array}$ & $97 \%+$ & $926-1386$ \\
\hline 70 & Virgibacillus halophilus & AB243851 & $\begin{array}{l}\text { CTGAAACTCAAAAGAATTGACG } \\
\text { AATACGTTCCCGGGCCTT }\end{array}$ & $94 \%+$ & $926-1386$ \\
\hline 71 & Virgibacillus sediminis & AY121430 & $\begin{array}{l}\text { CTGAAACTCAAAAGAATTGACG } \\
\text { AATACGTTCCCGGGCCTT }\end{array}$ & 96\%- & $946-1406$ \\
\hline 72 & Virgibacillus xinjiangensis & DQ664543 & $\begin{array}{l}\text { CTGAAACTCAAAAGAATTGACG } \\
\text { AATACGTTCCCGGGCCTT }\end{array}$ & $96 \%-$ & 894-1354 \\
\hline 73 & Virgibacillus chiguensis & EF101168 & $\begin{array}{l}\text { CTGAAACTCAAAAGAATTGACG } \\
\text { AATACGTTCCCGGGCCTT }\end{array}$ & $96 \%-$ & 919-1375 \\
\hline 74 & Virgibacillus dokdonensis & AY822043 & $\begin{array}{l}\text { CTGAAACTCAAAAGAATTGACG } \\
\text { AATACGTTCCCGGGTCTT }\end{array}$ & $96 \%-$ & $927-1383$ \\
\hline 75 & Virgibacillus carmonensis & AJ316302 & $\begin{array}{l}\text { CTGAAACTCAAAAGAATTGACG } \\
\text { AATACGTTCCCGGGCCTT }\end{array}$ & $95 \%+$ & $925-1385$ \\
\hline 76 & Virgibacillus necropolis & AJ315056 & $\begin{array}{l}\text { CTGAAACTCAAAAGAATTGACG } \\
\text { AATACGTTCCCGGGCCTT }\end{array}$ & $95 \%+$ & $925-1385$ \\
\hline 77 & Virgibacillus arcticus & EF675742 & $\begin{array}{l}\text { CTGAAACTCAAAAGAATTGACG } \\
\text { AATACGTTCCCGGGCCTT }\end{array}$ & $95 \%+$ & 809- 1269 \\
\hline
\end{tabular}


Table $2 \%$ similarity of 463 bp sequence of 16S rRNA gene of type sp. (Bacillus subtilis) with 16S rRNA sequences of different AEFB strains (downloaded from NCBI), primer sequences in these AEFB strains, presence and absence of restriction enzyme site and position of specific fragment in AEFB strains (Continued)

\begin{tabular}{|c|c|c|c|c|c|}
\hline 78 & Virgibacillus byunsanensis & FJ357159 & $\begin{array}{l}\text { CTGAAACTCAAAAGAATTGACG } \\
\text { AATACGTTCCCGGGCCTT }\end{array}$ & $95 \%+$ & $923-1383$ \\
\hline 79 & Virgibacillus salinus & FM205010 & $\begin{array}{l}\text { CTGAAACTCAAAAGAATTGACG } \\
\text { AATACGTTCCCGGGCCTT }\end{array}$ & $95 \%+$ & $932-1392$ \\
\hline 80 & Virgibacillus subterraneus & FJ746573 & $\begin{array}{l}\text { AATACGTTCCCGGCCCTT } \\
\text { CTGAAACTCAAAAGAATTGACG }\end{array}$ & $91 \%+$ & $905-1362$ \\
\hline 81 & Virgibacillus kekensis & AY121439 & $\begin{array}{l}\text { CTGAAACTCAAAAGAATTGACG } \\
\text { AATACGTTCCCGGGCCTT }\end{array}$ & $95 \%+$ & $945-1405$ \\
\hline 82 & $\begin{array}{l}\text { Geobacillus stearothermophilus } \\
\text { type sp. }\end{array}$ & AB021196, & $\begin{array}{l}\text { CTGAAACTCAAAGGAATTGACG } \\
\text { AATACGTTCCCGGGCCTT }\end{array}$ & $93 \%+$ & $912-1376$ \\
\hline 83 & Geobacillus kaustophilus & X60618 & $\begin{array}{l}\text { CTGAAACTCAAAGGAATTGACG } \\
\text { AATACGTTCCCGGGCCTT }\end{array}$ & $92 \%+$ & $933-1390$ \\
\hline 84 & Geobacillus lituanicus & AY044055 & $\begin{array}{l}\text { CTGAAACTCAAAGGAATTGACG } \\
\text { AATACGTTCCCGGGCCTT }\end{array}$ & $92 \%+$ & 933-1397 \\
\hline 85 & Geobacillus thermoleovorans & Z26923 & $\begin{array}{l}\text { CTGAAACTCAAAGGAATTGACG } \\
\text { AATACGTTCCCGGGCCTT }\end{array}$ & $93 \%+$ & $918-1382$ \\
\hline 86 & Geobacillus thermocatenulatus & AY608935 & $\begin{array}{l}\text { CTGAAACTCAAAGGAATTGACG } \\
\text { AATACGTTCCCGGGCCTT }\end{array}$ & $93 \%+$ & $940-1404$ \\
\hline 87 & Geobacillus jurassicus & AY312404 & $\begin{array}{l}\text { CTGAAACTCAAAGGAATTGACG } \\
\text { AATACGTTCCCGGGCCTT }\end{array}$ & $93 \%+$ & $915-1379$ \\
\hline 88 & Geobacillus uzenensis & AF276304 & $\begin{array}{l}\text { CTGAAACTCAAAGGAATTGACG } \\
\text { AATACGTTCCCGGGCCTT }\end{array}$ & $92 \%+$ & $907-1370$ \\
\hline 89 & Geobacillus subterraneus & AF276306 & $\begin{array}{l}\text { CTGAAACTCAAAGGAATTGACG } \\
\text { AATACGTTCCCGGGCCTT }\end{array}$ & $93 \%+$ & $931-1395$ \\
\hline 90 & Geobacillus thermodenitrificans & AY608961 & $\begin{array}{l}\text { CTGAAACTCAAAGGAATTGACG } \\
\text { AATACGTTCCCGGGCCTT }\end{array}$ & $93 \%+$ & $939-1409$ \\
\hline 91 & Geobacillus debilis & AJ564616 & $\begin{array}{l}\text { AATACGTTCTCGGGCCTT } \\
\text { CTGAAACTCAAAGGAATTGACG }\end{array}$ & $91 \%-$ & $936-1398$ \\
\hline 92 & Geobacillus toebii & AF326278 & $\begin{array}{l}\text { CTGAAACTCAAAGGAATTGACG } \\
\text { AATACGTTCCCGGGCCTT }\end{array}$ & $93 \%+$ & $910-1374$ \\
\hline 93 & Geobacillus thermoglucosidasius & AY608981 & $\begin{array}{l}\text { CTGAAACTCAAAGGAATTGACG } \\
\text { AATACGTTCCCGGGCCTT }\end{array}$ & $93 \%+$ & $939-1405$ \\
\hline 94 & Geobacillus caldoxylosilyticus & AF067651 & $\begin{array}{l}\text { CTGAAACTCAAAGGAATTGACG } \\
\text { AATACGTTCCCGGGCCTT }\end{array}$ & $94 \%+$ & $925-1389$ \\
\hline 95 & Geobacillus tepidamans & AY563003 & $\begin{array}{l}\text { GTGAAACTCAAAGGAATTGACG } \\
\text { AATACGTTCCCGGGCCTT }\end{array}$ & $95 \%+$ & $872-1334$ \\
\hline 96 & Geobacillus vulcani & AJ293805 & $\begin{array}{l}\text { CTGAAACTCAAAGGAATTGACG } \\
\text { AATACGTTCCCGGGCCTT }\end{array}$ & $93 \%+$ & $920-1384$ \\
\hline 97 & Filobacillus milosensis & AJ238042, & $\begin{array}{l}\text { CTGAAACTCAAAGGAATTGACG } \\
\text { AATACGTTCCCGGGCCTT }\end{array}$ & $94 \%+$ & $915-1375$ \\
\hline 98 & Jeotgalibacillus alimentarius. & AF281158 & $\begin{array}{l}\text { CTGAAACTCAAAGGAATTGACG } \\
\text { AATACGTTCCCGGGCCTT }\end{array}$ & $96 \%+$ & $911-1373$ \\
\hline 99 & Jeotgalibacillus salarius & EU874389 & $\begin{array}{l}\text { CTGAAACTCAAAGGAATTGACG } \\
\text { AATACGTTCCCGGGCCTT }\end{array}$ & $96 \%+$ & $910-1372$ \\
\hline 100 & Jeotgalibacillus campisalis & AY190535 & $\begin{array}{l}\text { CTGAAACTCAAAGGAATTGACG } \\
\text { AATACGTTCCCGGGCCTT }\end{array}$ & $95 \%+$ & $908-1370$ \\
\hline 101 & Jeotgalibacillus marinus & AJ237708 & $\begin{array}{l}\text { CTGAAACTCAAAGGAATTGACG } \\
\text { AATACGTTCCCGGGCCTT }\end{array}$ & $95 \%+$ & $918-1380$ \\
\hline 102 & Ureibacillus thermosphaericus & AB101594 & $\begin{array}{l}\text { CTGAAACTCAAAGGAATTGACG } \\
\text { AATACGTTCCCGGGCCTT }\end{array}$ & $92 \%+$ & $931-1392$ \\
\hline 103 & Ureibacillus composti & DQ348071 & $\begin{array}{l}\text { CTGAAACTCAAAGGAATTGACG } \\
\text { AATACGTTCCCGGGCCTT }\end{array}$ & $92 \%+$ & $929-1390$ \\
\hline
\end{tabular}


Table $2 \%$ similarity of 463 bp sequence of 16S rRNA gene of type sp. (Bacillus subtilis) with 16S rRNA sequences of different AEFB strains (downloaded from NCBI), primer sequences in these AEFB strains, presence and absence of restriction enzyme site and position of specific fragment in AEFB strains (Continued)

\begin{tabular}{|c|c|c|c|c|c|}
\hline 104 & Ureibacillus thermophilus & DQ348072 & $\begin{array}{l}\text { CTGAAACTCAAAGGAATTGACG } \\
\text { AATACGTTCCCGGGCCTT }\end{array}$ & $91 \%+$ & $931-1392$ \\
\hline 105 & Ureibacillus suwonensis & AY850379 & $\begin{array}{l}\text { CTGAAACTCAAAGGAATTGACG } \\
\text { AATACGTTCCCGGGCCTT }\end{array}$ & $92 \%-$ & $918-1379$ \\
\hline 106 & Ureibacillus terrenus & AJ276403 & $\begin{array}{l}\text { CTGAAACTCAAAGGAATTGACG } \\
\text { AATACGTTCCCGGGCCTT }\end{array}$ & $92 \%-$ & $900-1361$ \\
\hline 107 & Lysinibacillus boronitolerans & AB199591 & $\begin{array}{l}\text { CTGAAACTCAAAGGAATTGACG } \\
\text { AATACGTTCCCGGGCCTT }\end{array}$ & $93 \%+$ & $898-1360$ \\
\hline 108 & Lysinibacillus xylanilyticus & FJ477040 & $\begin{array}{l}\text { CTGAAACTCAAAGGAATTGACG } \\
\text { AATACGTTCCCGGGCCTT }\end{array}$ & $93 \%+$ & $826-1288$ \\
\hline 109 & Lysinibacillus fusiformis & AJ310083 & $\begin{array}{l}\text { CTGAAACTCAAAGGAATTGACG } \\
\text { AATACGTTCCCGGGCCTT }\end{array}$ & $93 \%+$ & $920-1382$ \\
\hline 110 & Lysinibacillus sphaericus & AJ310084 & $\begin{array}{l}\text { CTGAAACTCAAAGGAATTGACG } \\
\text { AATACGTTCCCGGGCCTT }\end{array}$ & $93 \%+$ & $920-1382$ \\
\hline 111 & Lysinibacillus parviboronicapiens & AB300598 & $\begin{array}{l}\text { CTGAAACTCAAAGGAATTGACG } \\
\text { AATACGTTCCCGGGCCTT }\end{array}$ & $93 \%+$ & $910-1372$ \\
\hline 112 & Terribacillus goriensis & DQ519571 & $\begin{array}{l}\text { CTGAAACTCAAAAGAATTGACG } \\
\text { AATACGTTCCCGGGCCTT }\end{array}$ & $94 \%+$ & $895-1355$ \\
\hline 113 & Terribacillus saccharophilus & AB243845 & $\begin{array}{l}\text { CTGAAACTCAAAAGAATTGACG } \\
\text { AATACGTTCCCGGGCCTT }\end{array}$ & $94 \%+$ & $922-1382$ \\
\hline 114 & Terribacillus halophilus & AB243849 & $\begin{array}{l}\text { CTGAAACTCAAAAGAATTGACG } \\
\text { AATACGTTCCCGGGCCTT }\end{array}$ & $95 \%+$ & $922-1382$ \\
\hline 115 & Terribacillus aidingensis & FJ386524 & $\begin{array}{l}\text { CTGAAACTCAAAAGAATTGACG } \\
\text { AATACGTTCCCGGGCCTT }\end{array}$ & $95 \%+$ & $922-1382$ \\
\hline 116 & Bacillus massiliensis & AY677116 & $\begin{array}{l}\text { AATACGTTCCCGGGCCTT } \\
\text { CTGAAACTCAAAGGAATTGACG }\end{array}$ & $93 \%+$ & $908-1370$ \\
\hline 117 & Bacillus cecembensis & AM773821 & $\begin{array}{l}\text { AATACGTTCCCGGGCCTT } \\
\text { CTGAAACTCAAAGGAATTGACG }\end{array}$ & $93 \%+$ & 931-1393 \\
\hline 118 & Bacillus odysseyi & AF526913 & $\begin{array}{l}\text { AATACGTTCCCGGGCCTT } \\
\text { CTGAAACTCAAAGGAATTGACG }\end{array}$ & $93 \%+$ & $931-1393$ \\
\hline 119 & Bacillus decisifrondis & DQ465405 & $\begin{array}{l}\text { AATACGTTCCCGGGCCTT } \\
\text { CTGAAACTCAAAGGAATTGACG }\end{array}$ & $85 \%+$ & $843-1305$ \\
\hline 120 & Bacillus psychrodurans & AJ277984 & $\begin{array}{l}\text { AATACGTTCCCGGGCCTT } \\
\text { CTGAAACTCAAAGGAATTGACG }\end{array}$ & 95\%- & $918-1380$ \\
\hline 121 & Bacillus psychrotolerans & AJ277983 & $\begin{array}{l}\text { AATACGTTCCCGGGCCTT } \\
\text { CTGAAACTCAAAGGAATTGACG }\end{array}$ & 95\%- & $903-1365$ \\
\hline 122 & Bacillus insolitus & AM980508 & $\begin{array}{l}\text { GAGGGGTTCCCGGGCCTT } \\
\text { CTGAAACTCAAAGGAATTGACG }\end{array}$ & $94 \%+$ & $917-1378$ \\
\hline 123 & Bacillus beijingensis & EF371374 & $\begin{array}{l}\text { AATACGTTCCCGGGTCTT } \\
\text { CTGAAACTCAAAGGAATTGACG }\end{array}$ & 96\%- & $929-1387$ \\
\hline 124 & Bacillus ginsengi & EF371375 & $\begin{array}{l}\text { AATACGTTCCCGGGTCTT } \\
\text { CTGAAACTCAAAGGAATTGACG }\end{array}$ & 97\%- & 929-1387 \\
\hline 125 & Bacillus aquimaris & AF483625 & $\begin{array}{l}\text { AATACGTTCCCGGGCCTT } \\
\text { CTGAAACTCAAAGGAATTGACG }\end{array}$ & $99 \%+$ & $910-1372$ \\
\hline 126 & Bacillus vietnamensis & AB099708 & $\begin{array}{l}\text { AATACGTTCCCGGGCCTT } \\
\text { CTGAAACTCAAAGGAATTGACG }\end{array}$ & $98 \%+$ & $903-1365$ \\
\hline 127 & Bacillus marisflavi & AF483624 & $\begin{array}{l}\text { AATACGTTCCCGGGCCTT } \\
\text { CTGAAACTCAAAGGAATTGACG }\end{array}$ & $100 \%+$ & $909-1371$ \\
\hline 128 & Bacillus seohaeanensis & AY667495 & $\begin{array}{l}\text { AATACGTTCCCGGGCCTT } \\
\text { CTGAAACTCAAAGGAATTGACG }\end{array}$ & $98 \%+$ & $872-1334$ \\
\hline 129 & Bacillus mycoides & AB021192 & $\begin{array}{l}\text { AATACGTTCCCGGGCCTT } \\
\text { CTGAAACTCAAAGGAATTGACG }\end{array}$ & $97 \%+$ & $907-1367$ \\
\hline
\end{tabular}


Table $2 \%$ similarity of 463 bp sequence of 16S rRNA gene of type sp. (Bacillus subtilis) with 16S rRNA sequences of different AEFB strains (downloaded from NCBI), primer sequences in these AEFB strains, presence and absence of restriction enzyme site and position of specific fragment in AEFB strains (Continued)

\begin{tabular}{|c|c|c|c|c|c|}
\hline 130 & Bacillus weihenstephanensis & AB021199 & $\begin{array}{l}\text { AATACGTTCCCGGGCCTT } \\
\text { CTGAAACTCAAAGGAATTGACG }\end{array}$ & $97 \%+$ & $925-1385$ \\
\hline 131 & Bacillus thuringiensis & D16281 & $\begin{array}{l}\text { AATACGTTCCCGGGCCTT } \\
\text { CTGAAACTCAAAGGAATTGACG }\end{array}$ & $97 \%+$ & 911-1371 \\
\hline 132 & Bacillus pseudomycoides & AF013121 & $\begin{array}{l}\text { CTGAAACTCAAAGGATTTGACG } \\
\text { AATACGTTCCCGGGCCTT }\end{array}$ & $95 \%+$ & $932-1392$ \\
\hline 133 & Bacillus funiculus & AB049195 & $\begin{array}{l}\text { CTGAAACTCAAAGGAATTGACG } \\
\text { AATACGTTCCCGGGCCTT }\end{array}$ & $98 \%+$ & 919-1379 \\
\hline 134 & Bacillus panaciterrae & AB245380 & $\begin{array}{l}\text { CTGAAACTCAAAGGAATTGACG } \\
\text { AATACGTTCCCGGGCCTT }\end{array}$ & $97 \%+$ & 904-1364 \\
\hline 135 & Bacillus flexus & AB021185 & $\begin{array}{l}\text { CTGAAACTCAAAGGAATTGACG } \\
\text { AATACGTTCCCGGGCCTT }\end{array}$ & $98 \%+$ & 923-1385 \\
\hline 136 & Bacillus megaterium & D16273 & $\begin{array}{l}\text { CTGAAACTCAAAGGAATTGACG } \\
\text { AATACGTTCCCGGGCCTT }\end{array}$ & $98 \%+$ & 910-1372 \\
\hline 137 & Bacillus koreensis & AY667496 & $\begin{array}{l}\text { CTGAAACTCAAAGGAATTGACG } \\
\text { AATACGTTCCCGGGCCTT }\end{array}$ & $96 \%+$ & 847-1309 \\
\hline 138 & Bacillus aerius & AJ831843 & $\begin{array}{l}\text { CTGAAACTCAAAGGAATTGACG } \\
\text { AATACGTTCCCGGGCCTT }\end{array}$ & $96 \%+$ & $922-1382$ \\
\hline 139 & Bacillus aerophilus & AJ831844 & $\begin{array}{l}\text { CTGAAACTCAAAGGAATTGACG } \\
\text { AATACGTTCCCGGGCCTT }\end{array}$ & $97 \%+$ & $927-1387$ \\
\hline 140 & Bacillus stratosphericus & AJ831841 & $\begin{array}{l}\text { CTGAAACTCAAAGGAATTGACG } \\
\text { AATACGTTCCCGGGCCTT }\end{array}$ & $97 \%+$ & $927-1387$ \\
\hline 141 & Bacillus sonorensis & AF302118 & $\begin{array}{l}\text { CTGAAACTCAAAGGAATTGACG } \\
\text { AATACGTTCCCGGGCCTT }\end{array}$ & $95 \%+$ & 908-1368 \\
\hline 142 & Bacillus amyloliquefaciens & AB255669 & $\begin{array}{l}\text { CTGAAACTCAAAGGAATTGACG } \\
\text { AATACGTTCCCGGGCCTT }\end{array}$ & $95 \%+$ & $909-1369$ \\
\hline 143 & Bacillus siamensis & GQ281299 & $\begin{array}{l}\text { CTGAAACTCAAAGGAATTGACG } \\
\text { AATACGTTCCCGGGCCTT }\end{array}$ & 95\%- & $931-1352$ \\
\hline 144 & Bacillus methylotrophicus & EU194897 & $\begin{array}{l}\text { CTGAAACTCAAAGGAATTGACG } \\
\text { AATACGTTCCCGGGCCTT }\end{array}$ & $95 \%+$ & 898-1358 \\
\hline 145 & Bacillus subtilis subsp. subtilis & AJ276351 & $\begin{array}{l}\text { CTGAAACTCAAAGGAATTGACG } \\
\text { AATACGTTCCCGGGCCTT }\end{array}$ & $95 \%+$ & 919-1379 \\
\hline 146 & Bacillus subtilis subsp. spizizenii & AF074970 & $\begin{array}{l}\text { CTGAAACTCAAAGGAATTGACG } \\
\text { AATACGTTCCCGGGCCTT }\end{array}$ & $95 \%+$ & $907-1367$ \\
\hline 147 & Bacillus vallismortis & AB021198 & $\begin{array}{l}\text { CTGAAACTCAAAGGAATTGACG } \\
\text { AATACGTTCCCGGGCCTT }\end{array}$ & $96 \%+$ & 924-1384 \\
\hline 148 & Bacillus mojavensis & AB021191 & $\begin{array}{l}\text { CTGAAACTCAAAGGAATTGACG } \\
\text { AATACGTTCCCGGGCCTT }\end{array}$ & $96 \%+$ & $920-1380$ \\
\hline 149 & Bacillus atrophaeus & AB021181 & $\begin{array}{l}\text { CTGAAACTCAAAGGAATTGACG } \\
\text { AATACGTTCCCGGGCCTT }\end{array}$ & $96 \%+$ & $909-1369$ \\
\hline 150 & Bacillus pumilus & AY876289 & $\begin{array}{l}\text { CTGAAACTCAAAGGAATTGACG } \\
\text { AATACGTTCCCGGGCCTT }\end{array}$ & $97 \%+$ & 879-1339 \\
\hline 151 & Bacillus safensis & AF234854 & $\begin{array}{l}\text { CTGAAACTCAAAGGAATTGACG } \\
\text { AATACGTTCCCGGGCCTT }\end{array}$ & $97 \%+$ & 879-1339 \\
\hline 152 & Bacillus altitudinis & AJ831842 & $\begin{array}{l}\text { CTGAAACTCAAAGGAATTGACG } \\
\text { AATACGTTCCCGGGCCTT }\end{array}$ & $97 \%+$ & 934-1394 \\
\hline 153 & Bacillus ginsengihumi & AB245378 & $\begin{array}{l}\text { TTGAAACTCAAAGGAATTGACG } \\
\text { AATACGTTCCCGGGCCTT }\end{array}$ & $98 \%-$ & 914-1376 \\
\hline 154 & Bacillus acidiproducens & EF379274 & $\begin{array}{l}\text { TTGAAACTCAAAGGAATTGACG } \\
\text { AATACGTTCCCGGGCCTT }\end{array}$ & 97\%- & 879-1341 \\
\hline 155 & Bacillus acidicola & AF547209 & $\begin{array}{l}\text { CTGAAACTCAAAGGAATTGACG } \\
\text { AATACGTTCCCGGGCCTT }\end{array}$ & $99 \%+$ & 934-1396 \\
\hline
\end{tabular}


Table $2 \%$ similarity of 463 bp sequence of 16S rRNA gene of type sp. (Bacillus subtilis) with 16S rRNA sequences of different AEFB strains (downloaded from NCBI), primer sequences in these AEFB strains, presence and absence of restriction enzyme site and position of specific fragment in AEFB strains (Continued)

\begin{tabular}{|c|c|c|c|c|c|}
\hline 156 & Bacillus oleronius & AY988598 & $\begin{array}{l}\text { CTGAAACTCAAAGGAATTGACG } \\
\text { AATACGTTCCCGGGCCTT }\end{array}$ & $97 \%+$ & 934-1396 \\
\hline 157 & Bacillus sporothermodurans & U49078 & $\begin{array}{l}\text { CTGAAACTCAAAGGAATTGACG } \\
\text { AATACGTTCCCGGGCCTT }\end{array}$ & $97 \%+$ & 904-1366 \\
\hline 158 & Bacillus carboniphilus & AB021182 & $\begin{array}{l}\text { CTGAAACTCAAAGGAATTGACG } \\
\text { AATACGTTCCCGGGCCTT }\end{array}$ & $95 \%+$ & 910-1372 \\
\hline 159 & Bacillus chungangensis & FJ514932 & $\begin{array}{l}\text { CTGAAACTCAAAGGAATTGACG } \\
\text { AATACGTTCCCGGGCCTT }\end{array}$ & $94 \%+$ & 890-1352 \\
\hline 160 & Bacillus endophyticus & AF295302 & $\begin{array}{l}\text { AATACGTTCCCGGGTCTT } \\
\text { CTGAAACTCAAAGGAATTGACG }\end{array}$ & $96 \%-$ & $906-1362$ \\
\hline 161 & Bacillus isabeliae & AM503357 & $\begin{array}{l}\text { CTGAAACTCAAAGGAATTGACG } \\
\text { AATACGTTCCCGGGCCTT }\end{array}$ & $98 \%+$ & $912-1372$ \\
\hline 162 & Bacillus shackletonii & AJ250318 & $\begin{array}{l}\text { CTGAAACTCAAAGGAATTGACG } \\
\text { AATACGTTCCCGGGCCTT }\end{array}$ & $98 \%+$ & 909-1371 \\
\hline 163 & Bacillus circulans & AY043084 & $\begin{array}{l}\text { CTGAAACTCAAAGGAATTGACG } \\
\text { AATACGTTCCCGGGCCTT }\end{array}$ & $96 \%+$ & $902-1364$ \\
\hline 164 & Bacillus nealsonii & EU656111 & $\begin{array}{l}\text { CTGAAACTCAAAGGAATTGACG } \\
\text { AATACGTTCCCGGGCCTT }\end{array}$ & $97 \%+$ & $928-1390$ \\
\hline 165 & Bacillus korlensis & EU603328 & $\begin{array}{l}\text { CTGAAACTCAAAGGAATTGACG } \\
\text { AATACGTTCCCGGGCCTT }\end{array}$ & $98 \%+$ & 889-1351 \\
\hline 166 & Bacillus siralis & AF071856 & $\begin{array}{l}\text { CTGAAACTCAAAGGAATTGACG } \\
\text { AATACGTTCCCGGGCCTT }\end{array}$ & $97 \%+$ & 905-1367 \\
\hline 167 & Bacillus benzoevorans & X60611 & $\begin{array}{l}\text { AATACGTTCCCGGGTCTT } \\
\text { CTGAAACTCAAAGGAATTGACG }\end{array}$ & 95\%- & $931-1386$ \\
\hline 168 & Bacillus firmus & D16268 & $\begin{array}{l}\text { CTGAAACTCAAAGGAATTGACG } \\
\text { AATACGTTCCCGGGCCTT }\end{array}$ & $97 \%+$ & $907-1369$ \\
\hline 169 & Bacillus infantis & AY904032 & $\begin{array}{l}\text { CTGAAACTCAAAGGAATTGACG } \\
\text { AATACGTTCCCGGGCCTT }\end{array}$ & $97 \%+$ & 871-1333 \\
\hline 170 & Bacillus oceanisediminis & GQ292772 & $\begin{array}{l}\text { CTGAAACTCAAAGGAATTGACG } \\
\text { AATACGTTCCCGGGCCTT }\end{array}$ & $97 \%+$ & $861-1323$ \\
\hline 171 & Bacillus kribbensis & DQ280367 & $\begin{array}{l}\text { CTGAAACTCAAAGGAATTGACG } \\
\text { AATACGTTCCCGGGCCTT }\end{array}$ & $96 \%-$ & 919-1381 \\
\hline 172 & Bacillus horneckiae & EU861362 & $\begin{array}{l}\text { TTGAAACTCAAAGGAATTGACG } \\
\text { AATACGTTCCCGGGCCTT }\end{array}$ & $98 \%+$ & $796-1258$ \\
\hline 173 & Bacillus badius & X77790 & $\begin{array}{l}\text { CTGAAACTCAAAGGAATTGACG } \\
\text { AATACGTTCCCGGGCCTT }\end{array}$ & 95\%- & $913-1370$ \\
\hline 174 & Bacillus smithii & Z26935 & $\begin{array}{l}\text { CTGAAACTCAAAGGAATTGACG } \\
\text { AATACGTTCCCGGGCCTT }\end{array}$ & $95 \%+$ & 924-1383 \\
\hline 175 & Bacillus aeolius & AJ504797 & $\begin{array}{l}\text { CTGAAACTCAAAGGAATTGACG } \\
\text { AATACGTTCCCGGGCCTT }\end{array}$ & 94\%- & $901-1361$ \\
\hline 176 & Bacillus coagulans & AB271752 & $\begin{array}{l}\text { CTGAAACTCAAAGGAATTGACG } \\
\text { AATACGTTCCCGGGCCTT }\end{array}$ & $96 \%-$ & $911-1373$ \\
\hline 177 & Bacillus alveayuensis & AY605232 & $\begin{array}{l}\text { CTGAAACTCAAAGGAATTGACG } \\
\text { AATACGTTCCCGGGCCTT }\end{array}$ & $95 \%+$ & 934-1396 \\
\hline 178 & Bacillus thermoamylovorans & L27478 & $\begin{array}{l}\text { CTGAAACTCAAAGGAATTGACG } \\
\text { AATACGTTCCCGGGCCTT }\end{array}$ & $92 \%+$ & 930-1391 \\
\hline 179 & Bacillus fordii & AY443039 & $\begin{array}{l}\text { CTGAAACTCAAAGGAATTGACG } \\
\text { AATACGTTCCCGGGCCTT }\end{array}$ & $94 \%+$ & $892-1354$ \\
\hline 180 & Bacillus fortis & AY443038 & $\begin{array}{l}\text { CTGAAACTCAAAGGAATTGACG } \\
\text { AATACGTTCCCGGGCCTT }\end{array}$ & $94 \%+$ & $927-1389$ \\
\hline 181 & Bacillus farraginis & AY443036 & $\begin{array}{l}\text { CTGAAACTCAAAGGAATTGACG } \\
\text { AATACGTTCCCGGGCCTT }\end{array}$ & $94 \%+$ & $838-1300$ \\
\hline
\end{tabular}


Table $2 \%$ similarity of 463 bp sequence of 16S rRNA gene of type sp. (Bacillus subtilis) with 16S rRNA sequences of different AEFB strains (downloaded from NCBI), primer sequences in these AEFB strains, presence and absence of restriction enzyme site and position of specific fragment in AEFB strains (Continued)

\begin{tabular}{|c|c|c|c|c|c|}
\hline 182 & Bacillus galactosidilyticus & AJ535638 & $\begin{array}{l}\text { CTGAAACTCAAAGGAATTGACG } \\
\text { AATACGTTCCCGGGCCTT }\end{array}$ & $95 \%+$ & 904-1367 \\
\hline 183 & Bacillus ruris & AJ535639 & $\begin{array}{l}\text { CTGAAACTCAAAGGAATTGACG } \\
\text { AATACGTTCCCGGGCCTT }\end{array}$ & 97\%- & $901-1363$ \\
\hline 184 & Bacillus lentus & AB021189 & $\begin{array}{l}\text { CTGAAACTCAAAGGAATTGACG } \\
\text { AATACGTTCCCGGGCCTT }\end{array}$ & $97 \%+$ & $928-1390$ \\
\hline 185 & Bacillus novalis & AJ542512 & $\begin{array}{l}\text { CTGAAACTCAAAGGAATTGACG } \\
\text { AATACGTTCCCGGGCCTT }\end{array}$ & $97 \%+$ & $908-1370$ \\
\hline 186 & Bacillus vireti & AJ542509 & $\begin{array}{l}\text { CTGAAACTCAAAGGAATTGACG } \\
\text { AATACGTTCCCGGGCCTT }\end{array}$ & $96 \%+$ & $908-1370$ \\
\hline 187 & Bacillus bataviensis & AJ542508 & $\begin{array}{l}\text { CTGAAACTCAAAGGAATTGACG } \\
\text { AATACGTTCCCGGGCCTT }\end{array}$ & $98 \%+$ & $908-1370$ \\
\hline 188 & Bacillus drentensis & AJ542506 & $\begin{array}{l}\text { CTGAAACTCAAAGGAATTGACG } \\
\text { AATACGTTCCCGGGCCTT }\end{array}$ & $98 \%+$ & 844-1306 \\
\hline 189 & Bacillus soli & AJ542513 & $\begin{array}{l}\text { CTGAAACTCAAAGGAATTGACG } \\
\text { AATACGTTCCCGGGCCTT }\end{array}$ & $97 \%+$ & $908-1370$ \\
\hline 190 & Bacillus fumarioli & AJ250056 & $\begin{array}{l}\text { CTGAAACTCAAAGGAATTGACG } \\
\text { AATACGTTCCCGGGCCTT }\end{array}$ & $95 \%+$ & 909-1371 \\
\hline 191 & Bacillus niacini & AB021194 & $\begin{array}{l}\text { CTGAAACTCAAAGGAATTGACG } \\
\text { AATACGTTCCCGGGCCTT }\end{array}$ & $98 \%+$ & $921-1383$ \\
\hline 192 & Bacillus pocheonensis & AB245377 & $\begin{array}{l}\text { CTGAAACTCAAAGGAATTGACG } \\
\text { AATACGTTCCCGGGCCTT }\end{array}$ & $98 \%+$ & $910-1372$ \\
\hline 193 & Bacillus boroniphilus & AB198719 & $\begin{array}{l}\text { CTGAAACTCAAAGGAATTGACG } \\
\text { AATACGTTCCCGGGCCTT }\end{array}$ & $97 \%+$ & 930-1392 \\
\hline 194 & Bacillus selenatarsenatis & AB262082 & $\begin{array}{l}\text { CTGAAACTCAAAGGAATTGACG } \\
\text { AATACGTTCCCGGGCCTT }\end{array}$ & $97 \%+$ & 870-1332 \\
\hline 195 & Bacillus jeotgali & AF221061 & $\begin{array}{l}\text { CTGAAACTCAAAGGAATTGACG } \\
\text { AATACGTTCCCGGGCCTT }\end{array}$ & $97 \%+$ & $908-1370$ \\
\hline 196 & Bacillus thioparans & DQ371431 & $\begin{array}{l}\text { CTGAAACTCAAAGGAATTGACG } \\
\text { AATACGTTCCCGGGCCTT }\end{array}$ & $97 \%+$ & $908-1370$ \\
\hline 197 & Bacillus foraminis & AJ717382 & $\begin{array}{l}\text { CTGAAACTCAAAGGAATTGACG } \\
\text { AATACGTTCCCGGGCCTT }\end{array}$ & $97 \%+$ & $922-1384$ \\
\hline 198 & Bacillus canaveralius & DQ870688 & $\begin{array}{l}\text { CTGAAACTCAAAGGAATTGACG } \\
\text { SEQUENEWAS SHORT }\end{array}$ & $97 \%$ & $887-1323$ \\
\hline 199 & Bacillus infernus & U20385 & $\begin{array}{l}\text { CTGAAACTCAAAGGAATTGACG } \\
\text { AATACGTTCCCGGGCCTN }\end{array}$ & $95 \%+$ & $921-1383$ \\
\hline 200 & Bacillus methanolicus & AB112727 & $\begin{array}{l}\text { CTGAAACTCAAAGGAATTGACG } \\
\text { AATACGTTCCCGGGCCTT }\end{array}$ & $96 \%+$ & $909-1372$ \\
\hline 201 & Bacillus butanolivorans & EF206294 & $\begin{array}{l}\text { CTGAAACTCAAAGGAATTGACG } \\
\text { AATACGTTCCCGGGCCTT }\end{array}$ & $96 \%+$ & $914-1380$ \\
\hline 202 & Bacillus simplex & AJ439078 & $\begin{array}{l}\text { CTGAAACTCAAAGGAATTGACG } \\
\text { AATACGTTCCCGGGCCTT }\end{array}$ & $98 \%+$ & 920-1379 \\
\hline 203 & Bacillus muralis & AJ316309 & $\begin{array}{l}\text { CTGAAACTCAAAGGAATTGACG } \\
\text { AATACGTTCCCGGGCCTT }\end{array}$ & $97 \%+$ & 909-1371 \\
\hline 204 & Bacillus psychrosaccharolyticus & AB021195 & $\begin{array}{l}\text { CTGAAACTCAAAGGAATTGACG } \\
\text { AATACGTTCCCGGGCCTT }\end{array}$ & $96 \%+$ & $900-1362$ \\
\hline 205 & Bacillus asahii & AB109209 & $\begin{array}{l}\text { CTGAAACTCAAAGGAATTGACG } \\
\text { AATACGTTCCCGGGCCTT }\end{array}$ & $96 \%+$ & $909-1373$ \\
\hline 206 & Bacillus indicus & AJ583158 & $\begin{array}{l}\text { CTGAAACTCAAAGGAATTGACG } \\
\text { AATACGTTCCCGGGCCTT }\end{array}$ & $96 \%+$ & 918-1381 \\
\hline 207 & Bacillus cibi & AY550276 & $\begin{array}{l}\text { CTGAAACTCAAAGGAATTGACG } \\
\text { AATACGTTCCCGGGCCTT }\end{array}$ & $97 \%+$ & 896-1358 \\
\hline
\end{tabular}


Table $2 \%$ similarity of 463 bp sequence of 16S rRNA gene of type sp. (Bacillus subtilis) with 16S rRNA sequences of different AEFB strains (downloaded from NCBI), primer sequences in these AEFB strains, presence and absence of restriction enzyme site and position of specific fragment in AEFB strains (Continued)

\begin{tabular}{|c|c|c|c|c|c|}
\hline 208 & Bacillus idriensis & AY904033 & $\begin{array}{l}\text { CTGAAACTCAAAGGAATTGACG } \\
\text { AATACGTTCCCGGGCCTT }\end{array}$ & $97 \%+$ & 889-1351 \\
\hline 209 & Bacillus niabensis & AY998119 & $\begin{array}{l}\text { CTGAAACTCAAAGGAATTGACG } \\
\text { AATACGTTCCCGGGCCTT }\end{array}$ & $95 \%+$ & $904-1366$ \\
\hline 210 & Bacillus fastidiosus & X60615 & $\begin{array}{l}\text { CTGAAACTCAAAGGAATTGACG } \\
\text { AATACGTTCCCGGGCCTT }\end{array}$ & $95 \%+$ & $930-1386$ \\
\hline 211 & Bacillus litoralis & AY608605 & $\begin{array}{l}\text { CTGAAACTCAAAGGAATTGACG } \\
\text { AATACGTTCCCGGGCCTT }\end{array}$ & $95 \%+$ & $908-1370$ \\
\hline 212 & Bacillus herbersteinensis & AJ781029 & $\begin{array}{l}\text { CTGAAACTCAAAGGAATTGACG } \\
\text { AATACGTTCCCGGGCCTT }\end{array}$ & $98 \%+$ & $908-1370$ \\
\hline 213 & Bacillus galliciensis & FM162181 & $\begin{array}{l}\text { CTGAAACTCAAAGGAATTGACG } \\
\text { AATACGTTCCCGGGCCTT }\end{array}$ & $96 \%+$ & $908-1370$ \\
\hline 214 & Bacillus alkalitelluris & AY829448 & $\begin{array}{l}\text { CTGAAACTCAAAGGAATTGACG } \\
\text { AATACGTTCCCGGGCCTT }\end{array}$ & $94 \%+$ & $911-1373$ \\
\hline 215 & Bacillus humi & AJ627210 & $\begin{array}{l}\text { CTGAAACTCAAAGGAATTGACG } \\
\text { AATACGTTCCCGGGCCTT }\end{array}$ & $96 \%$ & $910-1372$ \\
\hline 216 & Bacillus halmapalus & X76447 & $\begin{array}{l}\text { CTGAAACTCAAAGGAATTGACG } \\
\text { AATACGTTCCCGGGCCTT }\end{array}$ & $98 \%+$ & $908-1370$ \\
\hline 217 & Bacillus horikoshii & AB043865 & $\begin{array}{l}\text { CTGAAACTCAAAGGAATTGACG } \\
\text { AATACGTTCCCGGGCCTT }\end{array}$ & $97 \%+$ & 929-1391 \\
\hline 218 & Bacillus cohnii & X76437 & $\begin{array}{l}\text { CTGAAACTCAAAGGAATTGACG } \\
\text { AATACGTTCCCGGGCCTT }\end{array}$ & $97 \%+$ & $910-1372$ \\
\hline 219 & Bacillus acidiceler & DQ374637 & $\begin{array}{l}\text { CTGAAACTCAAAGGAATTGACG } \\
\text { AATACGTTCCCGGGCCTT }\end{array}$ & $97 \%+$ & $916-1376$ \\
\hline 220 & Bacillus luciferensis & AJ419629 & $\begin{array}{l}\text { CTGAAACTCAAAGGAATTGACG } \\
\text { AATACGTTCCCGGGCCTT }\end{array}$ & $97 \%+$ & $909-1369$ \\
\hline 221 & Bacillus azotoformans & AB363732 & $\begin{array}{l}\text { CTGAAACTCAAAGGAATTGACG } \\
\text { AATACGTTCCCGGGCCTT }\end{array}$ & $97 \%+$ & $909-1370$ \\
\hline 222 & Bacillus taeanensis & AY603978 & $\begin{array}{l}\text { CTGAAACTCAAAGGAATTGACG } \\
\text { AATACGTTCCCGGGCCTT }\end{array}$ & $97 \%+$ & $917-1378$ \\
\hline 223 & Bacillus macauensis & AY373018 & $\begin{array}{l}\text { CTGAAACTCAAAGGAATTGACG } \\
\text { AATACGTTCCCGGGCCTT }\end{array}$ & $95 \%+$ & $88-1350$ \\
\hline 224 & Bacillus rigui & EU939689 & $\begin{array}{l}\text { CTGAAACTCAAAGGAATTGACG } \\
\text { AATACGTTCCCGGGCCTT }\end{array}$ & 96\%- & $903-1365$ \\
\hline 225 & Bacillus solisalsi & EU046268 & $\begin{array}{l}\text { CTGAAACTCAAAAGGAATTGACG } \\
\text { AATACGTTCCCGGGCCTT }\end{array}$ & $95 \%-$ & $887-1349$ \\
\hline 226 & Bacillus gelatini & AJ551329 & $\begin{array}{l}\text { TTGAAACTCAAAGGAATTGACG } \\
\text { AATACGTTCCCGGGCCTT }\end{array}$ & $95 \%+$ & $909-1371$ \\
\hline 227 & Bacillus arsenicus & AJ606700 & $\begin{array}{l}\text { TTGAAACTCAAAGGAATTGACG } \\
\text { AATACGTTCCCGGGCCTT }\end{array}$ & 94\%- & $928-1390$ \\
\hline 228 & Bacillus barbaricus & AJ422145 & $\begin{array}{l}\text { TTGAAACTCAAAGGAATTGACG } \\
\text { AATACGTTCCCGGGCCTT }\end{array}$ & 96\%- & $882-1342$ \\
\hline 229 & Bacillus algicola & AY228462 & $\begin{array}{l}\text { TTGAAACTCAAAGGAATTGACG } \\
\text { AATACGTTCCCGGGCCTT }\end{array}$ & $97 \%+$ & $931-1393$ \\
\hline 230 & Bacillus hwajinpoensis & AF541966 & $\begin{array}{l}\text { TTGAAACTCAAAGGAATTGACG } \\
\text { AATACGTTCCCGGGCCTT }\end{array}$ & $96 \%+$ & $909-1371$ \\
\hline 231 & Bacillus decolorationis & AJ315075 & $\begin{array}{l}\text { TTGAAACTCAAAGGAATTGACG } \\
\text { AATACGTTCCCGGGCCTT }\end{array}$ & $94 \%+$ & $909-1371$ \\
\hline 232 & Bacillus okuhidensis & AB047684 & $\begin{array}{l}\text { TTGAAACTCAAAGGAATTGACG } \\
\text { AATACGTTCCCGGGCCTT }\end{array}$ & $96 \%+$ & 874-1335 \\
\hline 233 & Bacillus lehensis & AY793550 & $\begin{array}{l}\text { AATACGTTCCCGGGTCTT } \\
\text { CTGAAACTCAAAGGAATTGACG }\end{array}$ & 95\%- & 939-1395 \\
\hline
\end{tabular}


Table $2 \%$ similarity of 463 bp sequence of 16S rRNA gene of type sp. (Bacillus subtilis) with 16S rRNA sequences of different AEFB strains (downloaded from NCBI), primer sequences in these AEFB strains, presence and absence of restriction enzyme site and position of specific fragment in AEFB strains (Continued)

\begin{tabular}{|c|c|c|c|c|c|}
\hline 234 & Bacillus oshimensis & AB188090 & $\begin{array}{l}\text { AATACGTTCCCGGGTCTT } \\
\text { CTGAAACTCAAAGGAATTGACG }\end{array}$ & 95\%- & $937-1393$ \\
\hline 235 & Bacillus patagoniensis & AY258614 & $\begin{array}{l}\text { AATACGTTCCCGGGTCTT } \\
\text { TTGAAACTCAAAGGAATTGACG }\end{array}$ & $95 \%-$ & $913-1369$ \\
\hline 236 & Bacillus clausii & X76440 & $\begin{array}{l}\text { AATACGTTCCCGGGTCTT } \\
\text { CTGAAACTCAAAGGAATTGACG }\end{array}$ & $95 \%-$ & $913-1369$ \\
\hline 237 & Bacillus gibsonii & X76446 & $\begin{array}{l}\text { CTGAAACTCAAAGGAATTGACG } \\
\text { AATACGTTCCCGGGCCTT }\end{array}$ & $95 \%+$ & $912-1372$ \\
\hline 238 & Bacillus murimartini & AJ316316 & $\begin{array}{l}\text { CTGAAACTCAAAGGAATTGACG } \\
\text { AATACGTTCCCGGGCCTT }\end{array}$ & $95 \%+$ & $913-1373$ \\
\hline 239 & Bacillus plakortidis & AJ880003 & $\begin{array}{l}\text { CTGAAACTCAAAGGAATTGACG } \\
\text { AATACGTTCCCGGGCCTT }\end{array}$ & $95 \%+$ & $906-1366$ \\
\hline 240 & Bacillus pseudalcaliphilus & X76449 & $\begin{array}{l}\text { CTGAAACTCAAAGGAATTGACG } \\
\text { AATACGTTCCCGGGCCTT }\end{array}$ & $96 \%+$ & $909-1371$ \\
\hline 241 & Bacillus trypoxylicola & AB434284 & $\begin{array}{l}\text { CTGAAACTCAAAGGAATTGACG } \\
\text { AATACGTTCCCGGGCCTT }\end{array}$ & $97 \%+$ & $911-1373$ \\
\hline 242 & Bacillus alcalophilus & X76436 & $\begin{array}{l}\text { CTGAAACTCAAAGGAATTGACG } \\
\text { AATACGTTCCCGGGCCTT }\end{array}$ & $96 \%+$ & $909-1371$ \\
\hline 243 & Bacillus bogoriensis & AY376312 & $\begin{array}{l}\text { CTGAAACTCAAAGGAATTGAGC } \\
\text { AATACGTTCCCGGGCCTT }\end{array}$ & $97 \%+$ & $911-1374$ \\
\hline 244 & Bacillus akibai & AB043858 & $\begin{array}{l}\text { TTGAAACTCAAAGGAATTGACG } \\
\text { AATACGTTCCCGGGCCTT }\end{array}$ & $96 \%+$ & $950-1411$ \\
\hline 245 & Bacillus krulwichiae & AB086897 & $\begin{array}{l}\text { TTGAAACTCAAAGGAATTGACG } \\
\text { AATACGTTCCCGGGCCTT }\end{array}$ & $94 \%+$ & $912-1374$ \\
\hline 246 & Bacillus okhensis & DQ026060 & $\begin{array}{l}\text { TTGAAACTCAAAGGAATTGACG } \\
\text { AATACGTTCCCGGGCCTT }\end{array}$ & $96 \%+$ & $916-1378$ \\
\hline 247 & Bacillus wakoensis & AB043851 & $\begin{array}{l}\text { TTGAAACTCAAAGGAATTGACG } \\
\text { AATACGTTCCCGGGCCTT }\end{array}$ & $95 \%+$ & $930-1392$ \\
\hline 248 & Bacillus hemicellulosilyticus & AB043846 & $\begin{array}{l}\text { TTGAAACTCAAAGGAATTGACG } \\
\text { AATACGTTCCCGGGCCTT }\end{array}$ & $96 \%+$ & $940-1402$ \\
\hline 249 & Bacillus macyae & AY032601cpf & $\begin{array}{l}\text { TTGAAACTCAAAGGAATTGACG } \\
\text { AATACGTTCCCGGGCCTT }\end{array}$ & $96 \%+$ & $916-1378$ \\
\hline 250 & Bacillus alkalinitrilicus & EF422411 & $\begin{array}{l}\text { CTGAAACTCAAAGGAATTGACG } \\
\text { AATACGTTCCCGGGCCTT }\end{array}$ & $95 \%+$ & $919-1381$ \\
\hline 251 & Bacillus pseudofirmus & X76439 & $\begin{array}{l}\text { TTGAAACTCAAAGGAATTGACG } \\
\text { AATACGTTCCCGGGCCTT }\end{array}$ & $97 \%+$ & $910-1372$ \\
\hline 252 & Bacillus qingdaonensis & DQ115802 & $\begin{array}{l}\text { TTGAAACTCAAAGGAATTGACG } \\
\text { AATACGTTCCCGGGCCTT }\end{array}$ & $93 \%+$ & $913-1375$ \\
\hline 253 & Bacillus halochares & AM982516 & $\begin{array}{l}\text { TTGAAACTCAAAGGAATTGACG } \\
\text { AATACGTTCCCGGGCCTT }\end{array}$ & 93\%- & $881-1343$ \\
\hline 254 & Bacillus aidingensis & DQ504377 & $\begin{array}{l}\text { TTGAAACTCAAAGGAATTGACG } \\
\text { AATACGTTCCCGGGCCTT }\end{array}$ & 93\%- & $946-1407$ \\
\hline 255 & Bacillus salarius & AY667494 & $\begin{array}{l}\text { TTGAAACTCAAAGGAATTGACG } \\
\text { AATACGTTCCCGGGCCTT }\end{array}$ & $93 \%+$ & $858-1320$ \\
\hline 256 & Bacillus persepolensis & FM244839 & $\begin{array}{l}\text { TTGAAACTCAAAGGAATTGACG } \\
\text { AATACGTTCCCGGGCCTT }\end{array}$ & $93 \%+$ & $940-1402$ \\
\hline 257 & Bacillus agaradhaerens & X76445 & $\begin{array}{l}\text { CTGAAACTCAAAGGAATTGACG } \\
\text { AATACGTTCCCGGGTCTT }\end{array}$ & $96 \%+$ & $925-1385$ \\
\hline 258 & Bacillus neizhouensis & EU925618 & $\begin{array}{l}\text { TTGAAACTCAAAGGAATTGACG } \\
\text { AATACGTTCCCGGGCCTT }\end{array}$ & $96 \%+$ & $905-1367$ \\
\hline 259 & Bacillus beveridgei & FJ825145 & $\begin{array}{l}\text { CTGAAACTCAAAGGAATTGACG } \\
\text { AATACGTTCCCGGGCCTT }\end{array}$ & $94 \%+$ & $944-1409$ \\
\hline
\end{tabular}


Table $2 \%$ similarity of 463 bp sequence of 16S rRNA gene of type sp. (Bacillus subtilis) with 16S rRNA sequences of different AEFB strains (downloaded from NCBI), primer sequences in these AEFB strains, presence and absence of restriction enzyme site and position of specific fragment in AEFB strains (Continued)

\begin{tabular}{|c|c|c|c|c|c|}
\hline 260 & Bacillus chagannorensis & AM492159 & $\begin{array}{l}\text { CTGAAACTCAAAGGAATTGACG } \\
\text { AATACGTTCCCGGGCCTT }\end{array}$ & $94 \%+$ & $945-1407$ \\
\hline 261 & Bacillus saliphilus & AJ493660 & $\begin{array}{l}\text { CTGAAACTCAAAGGAATTGACG } \\
\text { AATACGTTCCCGGGCCTT }\end{array}$ & $93 \%+$ & 919-1381 \\
\hline 262 & Bacillus aurantiacus & AJ605773 & $\begin{array}{l}\text { CTGAAACTCAAAGGAATTGACG } \\
\text { AATACGTTCCCGGGCCTT }\end{array}$ & $95 \%+$ & 929-1381 \\
\hline 263 & Bacillus vedderi & Z48306 & $\begin{array}{l}\text { CTGAAACTCAAAGGAATTGACG } \\
\text { AATACGTTCCCGGGCCTT }\end{array}$ & 95\%- & $905-1367$ \\
\hline 264 & Bacillus cellulosilyticus & AB043852 & $\begin{array}{l}\text { CTGAAACTCAAAGGAATTGACG } \\
\text { AATACGTTCCCGGGCCTT }\end{array}$ & 95\%- & $924-1386$ \\
\hline 265 & Bacillus clarkii & X76444 & $\begin{array}{l}\text { CTGAAACTCAAAGGAATTGACG } \\
\text { AATACGTTCCCGGGCCTT }\end{array}$ & $95 \%+$ & $926-1328$ \\
\hline 266 & Bacillus polygoni & AB292819 & $\begin{array}{l}\text { CTGAAACTCAAAGGAATTGACG } \\
\text { AATACGTTCCCGGGCCTT }\end{array}$ & 94\%- & $945-1408$ \\
\hline 267 & Bacillus horti & D87035 & $\begin{array}{l}\text { CTGAAACTCAAAGGAATTGACG } \\
\text { AATACGTTCCCGGGCCTT }\end{array}$ & $93 \%+$ & 923-1378 \\
\hline \multirow[t]{2}{*}{268} & Bacillus mannanilyticus & AB043864 & $\begin{array}{l}\text { AATACGTTCCCGGGTCTT } \\
\text { CTGAAACTCAAAGGAATTGACG }\end{array}$ & 96\%- & $955-1413$ \\
\hline & $\begin{array}{l}\text { Actinobacteria (High GC content } \\
\text { gram positive bacteria) }\end{array}$ & & & & \\
\hline 269 & Corynebacterium diphtheriae & X84248 & $\begin{array}{l}\text { CTAAAACTCAAAGGAATTGACG } \\
\text { AATACGTNCCCGGGCCTT }\end{array}$ & $83 \%-$ & 880-1341 \\
\hline 270 & Mycobacterium tuberculosis & X58890 & $\begin{array}{l}\text { CTAAAACTCAAAGGAATTGACG } \\
\text { AATACGTTCCCGGGCCTT }\end{array}$ & $85 \%-$ & $1541-2002$ \\
\hline 271 & Nocardia asteroides & AF430019 & $\begin{array}{l}\text { CTAAAACTCAAAGGAATTGACG } \\
\text { AATACGTTCCCGGGCCTT }\end{array}$ & $84 \%-$ & $875-1376$ \\
\hline \multirow[t]{2}{*}{272} & $\begin{array}{l}\text { Streptomyces lavendulae subsp. } \\
\text { lavendulae }\end{array}$ & D85116 & $\begin{array}{l}\text { CTAAAACTCANAGGAATTGACG } \\
\text { AATACGTTCCCGGGCCTT }\end{array}$ & $81 \%-$ & $893-1361$ \\
\hline & $\begin{array}{l}\text { Low GC content Firmicutes } \\
\text { (gram +ve) }\end{array}$ & & & & \\
\hline 273 & Staphylococcus chromogenes & D83360 & $\begin{array}{l}\text { AATACGTTCCCGGGTCTT } \\
\text { CTGAAACTCAAAGGAATTGACG }\end{array}$ & $92 \%+$ & $913-1371$ \\
\hline 274 & Streptococcus pyogenes & AB002521 & $\begin{array}{l}\text { TTGAAACTCAAAGGAATTGACG } \\
\text { AATACGTTCCCGGGCCTT }\end{array}$ & $89 \%+$ & $890-1350$ \\
\hline 275 & Enterococcus faecalis & $\mathrm{AB} 012212$ & $\begin{array}{l}\text { TTGAAACTCAAAGGAATTGACG } \\
\text { AATACGTTCCCGGGCCTT }\end{array}$ & $91 \%+$ & 939-1395 \\
\hline 276 & Clostridium populeti & X71853 & $\begin{array}{l}\text { ATGAAACTCAAAGGAATTGACG } \\
\text { AATACGTTCCCGGGTCIT }\end{array}$ & $86 \%-$ & 903-1359 \\
\hline \multirow[t]{2}{*}{277} & Listeria monocytogenes. & $\times 56153$ & $\begin{array}{l}\text { AATACGTTCCCGGGCCTN } \\
\text { TTGAAACTCAAAGGAATTGACG }\end{array}$ & $94 \%+$ & 936-1392 \\
\hline & Alpha proteobacteria & & & & \\
\hline 278 & Rhizobium leguminosarum & U29386 & $\begin{array}{l}\text { TTAAAACTCAAAGGAATTGACG } \\
\text { AATACGTTCCCGGGCCTT }\end{array}$ & $86 \%-$ & $913-1371$ \\
\hline 279 & Azospirillum lipoferum & Z29619 & $\begin{array}{l}\text { TTAAAACTCAAAGGAATTGACG } \\
\text { AATACGTTCCCGGGCCTT }\end{array}$ & $84 \%-$ & $845-1305$ \\
\hline \multirow[t]{2}{*}{280} & Acetobacterium woodii & X96954 & $\begin{array}{l}\text { TTGAAACTCAAAGGAATTGACG } \\
\text { AATGCGTTCCCGGGTCTT }\end{array}$ & $90 \%-$ & 840-1305 \\
\hline & Beta proteobacteria & & & & \\
\hline 281 & Burkholderia cepacia & U96927 & $\begin{array}{l}\text { AATACGTTCCCGGGTCTT } \\
\text { TTAAAACTCAAAGGAATTGACG }\end{array}$ & $82 \%-$ & 870-1322 \\
\hline
\end{tabular}


Table $2 \%$ similarity of 463 bp sequence of 165 rRNA gene of type sp. (Bacillus subtilis) with $16 \mathrm{~S}$ rRNA sequences of different AEFB strains (downloaded from NCBI), primer sequences in these AEFB strains, presence and absence of restriction enzyme site and position of specific fragment in AEFB strains (Continued)

\begin{tabular}{|c|c|c|c|c|c|}
\hline \multirow[t]{2}{*}{282} & Bordetella pertussis & U04950 & $\begin{array}{l}\text { TTAAAACTCAAAGGAATTGACG } \\
\text { AATACGTTCCCGGGTCTT }\end{array}$ & $81 \%-$ & $922-1375$ \\
\hline & Gamma proteobacteria & & & & \\
\hline 283 & Pseudomonas aeruginosa & X06684 & $\begin{array}{l}\text { AATACGTCCCCGGGCCTT } \\
\text { TTAAAACTCAAATGAATTGACG }\end{array}$ & $86 \%-$ & $923-1384$ \\
\hline 284 & Escherichia coli & X80725 & $\begin{array}{l}\text { TTAAAACTCAAATGAATTGACG } \\
\text { AATACGTTCCCGGGTCTT }\end{array}$ & $83 \%-$ & $921-1379$ \\
\hline 285 & $\begin{array}{l}\text { Klebsiella pneumoniae subsp. } \\
\text { pneumoniae }\end{array}$ & X87276 & $\begin{array}{l}\text { TTAAAACTCAAATGAATTGACG } \\
\text { AATACGTTCCCGGGTCTT }\end{array}$ & $82 \%-$ & $920-1381$ \\
\hline 286 & Shigella dysenteriae & X96966 & $\begin{array}{l}\text { TTAAAACTCAAATGAATTGACG } \\
\text { AATACGTTCCCGGGTCIT }\end{array}$ & $83 \%-$ & $908-1362$ \\
\hline
\end{tabular}

aryabhattai have not shown any grouping with any other strain. Second dendrogram (Figure 2b) containing 29 different closely related species has been divided in to two major clusters and only one species Bacillus siamensis GQ281299 has not shown any grouping with any other member. 7 bacterial species i.e. Bacillus aquaemaris AF483625, Bacillus marisflavi AF483624, Bacillus seohaeanensis AY667495, Bacillus vietnamensis AB099708, Bacillus flexus AB021185, Bacillus megaterium D16273, Bacillus koreensis AY667496 lie in one cluster. Other, 21 bacterial strains have shared the other major cluster.

\section{Discussion}

When we use molecular approaches to study microbial communities then the use of universal primers is not fully successful in finding the clear picture of community. Various researchers have faced such type of problems as Van Elsas et al. (2002) faced the problem when they studied two 16S rDNA clone libraries (one from grassland and one from arable land) prepared with bacterial primers and most of the isolated strains were found to be belonging to phylum Proteobacteria and the number of clones related to Bacilli were very few. When the same microbial communities were studied by Garbeva et al. (2003), by using Bacillus specific primers then a lot of Bacillus clones were isolated. The problem faced by universal primers can be overcome by the use of group specific primers and various researchers have used group specific primers in their studies to overcome this problem. Heuer and Smalla (1997) used Actinomycete specific primers to monitor Actinomycete communities in the potato rhizosphere. Similarly Boon et al. (2001) used several group specific nested PCR systems to identify a lot of groups under same DGGE conditions. So the need of group specific primers is there to find out the diversity and identity of the members of a specific group.

In the present research we have focused on identification and classification of AEFB by using a specific fragment of $16 \mathrm{~S}$ rRNA gene. So in the following session we have discussed the research related to identification and classification of bacilli by using $16 \mathrm{~S}$ rRNA gene. As Many researchers have developed a lot of different Bacillus specific primers i.e. Goto et al. (2000), synthesized a Bacillus specific prime pair which was used to amplify a $275 \mathrm{bp}$ sequence near the 5 ' end of $16 \mathrm{~S}$ rDNA gene and this sequence was very specific for identification and classification of Bacillus strains. Garbeva et al. (2003) developed a Bacillus specific primer pair (Bac F and Bac R). Specificity of both primers was checked independently and some species of Bacillus and other related genera have shown $100 \%$ similarity with primer Bac F and likewise the reverse primer has shown similarity with 31 different species of Bacillus and related genera. Vardhan et al. (2011) developed a primer pair specific for amplification of a hyper variable region in $16 \mathrm{~S}$ rDNA gene of Bacillus and related genera.

In the present study we found that a restriction digestion product of $16 \mathrm{~S}$ rRNA gene (460 bp) by HaeIII enzyme was specific for Bacillus and related genera. Position of this fragment was near the 3 ' end of $16 \mathrm{~S}$ rDNA gene and primer pair specific to this $463 \mathrm{bp}$ fragment has been designed. Primer pair when checked for specificity has shown amplification of a $463 \mathrm{bp}$ long fragment in strains belonging to genera Bacillus, Lysinibacillus, Terribacillus, Brevibacillus and Jeotgalibacillus. No any amplification was seen in two AEFB strains i.e. Bacillus arsenicus and Paenibacillus taiwanensis and 9 different strains of bacterial lineages other than AEFB (Figure 1c\&d). Reason for no amplification of this fragment in Bacillus arsenicus and Paenibacillus taiwanensis is may be due to the reason that during the course of evolution these have faced some variations because of which the restriction enzyme sites for Hae III enzymes were deleted at that position and primer pair designed in the present study includes the restriction site which causes the non specificity for primer.

Results of our study indicate that primer pair designed here is specific for Bacillus and related genera and not for other bacterial lineages. Primer pair when checked for 

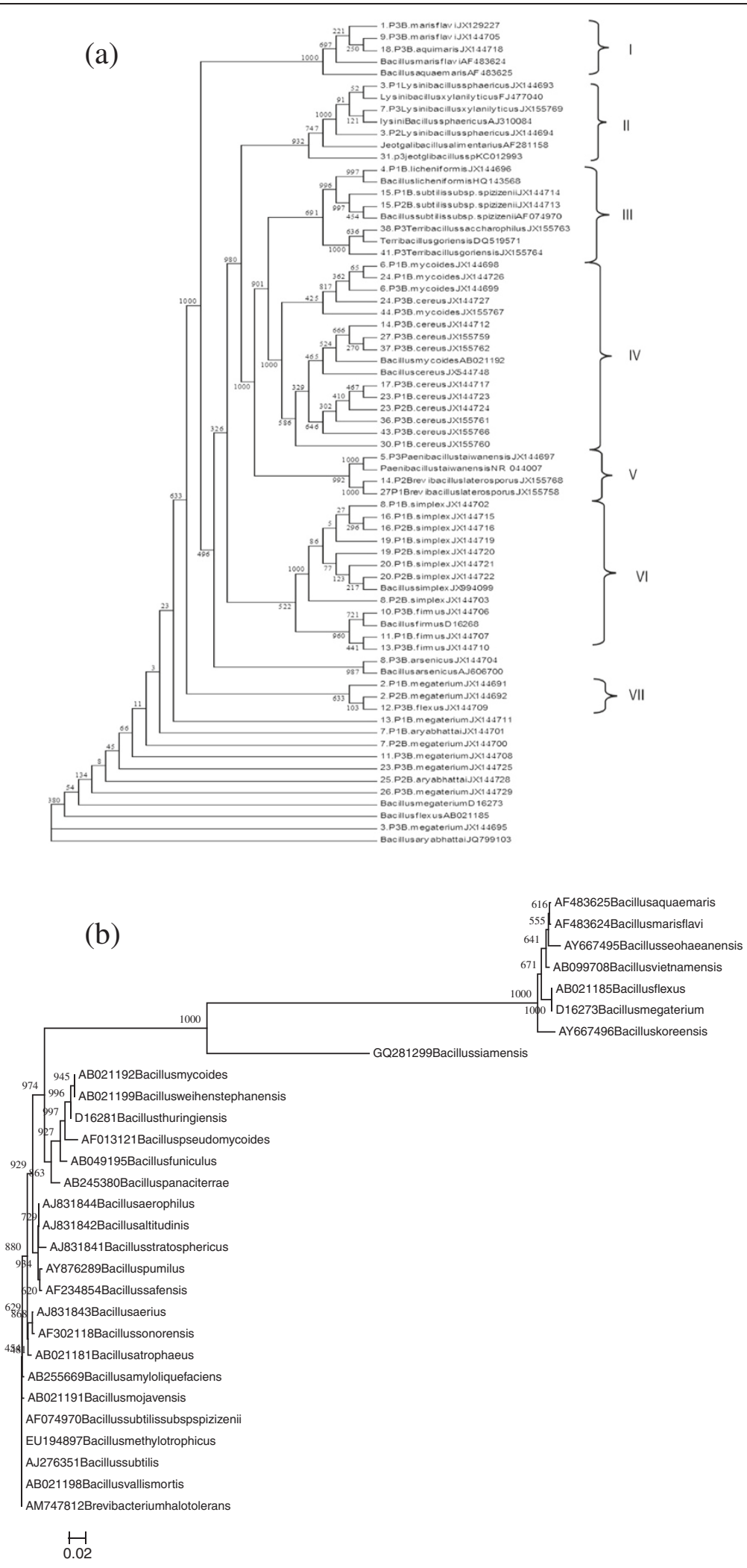

Figure 2 Dendrograms showing the phylogenetic relationship (a) 52 AEFB strains with reference sequences (b) 29 closely related species of genera Bacillus based on 463 bp long 16S rRNA sequences. 
homology (in silico) has shown 100\% homology with $16 \mathrm{~S}$ rDNA sequences of 120 species related to genera Bacillus. Bacillus species which do not have shown 100\% similarity of these primers have acquired anomalous positions in the classification based on 16S rRNA gene (Yarza et al. 2010). While some species i.e. B. pseudomycoides AF013121, B. ginsengihumi AB245378, B. acidiproducens EF379274, B. endophyticus, AF295302, B. benzoevorans, X60611, B. horneckiae EU86136 have shown anomalous positions with other bacterial lineages according to classification systems based on 16S rRNA gene (Yarza et al. 2010) in spite of having homology with primer pair in our study. Bacilli strains other than the genus Bacillus have also shown the primer pair similarity and these genera are Virgibacillus (7), Geobacillus (5), Filobacillus (1), Jeotgalibacillus (4) and Ureibacillus (5). Almost all the species checked for primer pair homology has shown 100\% similarity except Virgibacillus pantothenticus D16275, Virgibacillus proomii and AJ012667. All of these genera belong to the family Bacillaceae except Jeotgalibacillus which belong to the family Planococcaceae. Bacillus related genera which don't have shown primer pair similarity are Alicyclobacillus (7), Amphibacillus (5), Aneurinibacillus (5), Brevibacillus (16), Gracilibacillus (9) and Paenibacillus (5). Only a few members of these genera have shown homology with primer pair and these are Alicyclobacillus acidocaldarius AJ496806, Alicyclobacillus tolerans Z21979, Brevibacillus invocatus AF378232, Brevibacillus panacihum. Genera which do not have shown primer specificity belong to different species other than Bacillaceae except Amphibacillus, Gracilibacillus and Terribacillus. In our study genera belonging to family Bacillaceae have shown primer specificity and genera belonging to family other than Bacillaceae have not shown primer specificity except some genera which have shown primer specificity in reverse order.

Phylogenetic relationship based on 463 bp sequence of 52 bacilli strains (taken in our study) along with reference sequences (downloaded from NCBI) (Figure 2a) has shown that different bacterial strains belonging to same species and genera have shared a single group except some strains belonging to Bacillus megaterium, $B$. aryabhattai and B. flexus. As strains belonging to species Bacillus megaterium have not grouped in one cluster. Out of total 8 strains of $B$. megaterium, only two strains belonging to species $B$. megaterium have made grouping with B. flexus. Another 6 strains of B. megaterium and two strains of $B$. aryabhattai have not shown any grouping with any other strain, however all these eight strains lie below B. megaterium and B. flexus group. This shows that different strains of $B$. megaterium and $B$. aryabhattai (close relative of B. megaterium) have remarkable strain to strain genetic variations. Grouping of strains belonging to Bacillus related genera in between the strains related to Bacillus indicates that during the course of evolution these genera have been evolved from the older one genera i.e. Bacillus which is similar to the classifications according to others (Xu and Cote 2003; Yarza et al. 2010; Vardhan et al. 2011). Further the phylogenetic relationship of some closely related strains of genera Bacillus, sharing a single cluster in the all species living tree (Yarza et al. 2010) have shown the same phylogenetic relationship in our study (Figure 2b). The only exception is Bacillus siamensis GQ281299 which has not shown any grouping with any other Bacillus species. However, in all species living tree this strain has shown relationship with other Bacillus species which lie in the lower cluster in our study (Figure 2b).

From the present study we can conclude that the restriction digestion of $16 \mathrm{~S}$ rRNA gene by Haell enzyme and amplification of $463 \mathrm{bp}$ fragment with specific primers designed in our study are easy methods for identification of Bacillus and related genera. Further the sequence information and multiple alignment of 463 bp fragment of Bacillus and related genera have been proved to be a good identification and classification tool for Bacillus and related genera.

\section{Competing interests \\ The authors declare that they have no competing interests.}

\section{Authors' contribution}

SK carried out experimental research work. MP has contributed in interpretation of data and preparation of manuscript. KS has participated in sequence alignment. JPY has supervised the research work, prepared and edited the manuscript. All authors read and approved the final manuscript.

\section{Acknowledgements}

Council of Scientific and industrial Research (CSIR), India is acknowledged to provide Senior Research Fellowship to author Sangeeta Kadyan. Authors are also thankful to Department of Science and Technology, New Delhi for providing financial grants under DST-FIST programme and UGC, New Delhi for financial grant under UGC-SAP scheme.

Received: 3 August 2013 Accepted: 30 October 2013

Published: 9 November 2013

\section{References}

Ash C, Farrow AE, Wallbanks S, Collins MD (1991) Phylogenetic heterogeneity of the genus Bacillus revealed by comparative analysis of small-subunit-ribosomal RNA sequences. Lett Appl Microbiol 13:202-206

Ash C, Priest FG, Collins D (1993) Molecular identification of rRNA group 3 bacilli (Ash, Farrow, Wallbanks and Collins) using a PCR probe test. Proposal for the creation of a new genus Paenibacillus. Antonie van Leeuwenhoek 64:253-260 Boon N, Windt W, Verstraete W, Top EM (2001) Evaluation of nested PCR-DGGE (denaturing gradient gel electropho-resis) with group-specific 165 rRNA primers for the analysis of bacterial communities from different waste water treatment plants. FEMS Microbiol Ecol 39:101-112

Daffonchio D, Borin S, Consolandi A, Mora D, Manachini PL et al (1998a) 16S-23S rRNA internal transcribed spacers as molecular markers for the species of the $16 S$ rRNA group I of the genus Bacillus. FEMS Microbiol Lett 163:229-236

Daffonchio D, Borin S, Frova G, Manachini PL, Sorlini C (1998b) PCR fingerprinting of whole genomes: the spacers between the $16 \mathrm{~S}$ and $23 \mathrm{~S}$ rRNA genes and of intergenic tRNA gene regions reveal a different intraspecific genomic variability of Bacillus cereus and Bacillus licheniformis. Int J Syst Bacteriol 48:107-116

De Clerck E, Van Mol K, Jannes G, Rossau R, de Vos P (2004) Design of a 50 exonuclease-based real-time PCR assay for simultaneous detection of Bacillus licheniformis, members of the 'B. cereus group' and B. fumarioli in gelatin. Lett Appl Microbiol 39:109-115 
Fortina MG, Pukall R, Schumann P, Mora D, Parini C et al (2001) Ureibacillus gen. nov., a new genus to accommodate Bacillus thermosphericus (Anderson et al. 1995) emendation of Ureibacillus thermosphericus and description of Ureibacillus terrreneus sp. nov. Int J Syst Evol Microbiol 51:447-455

Garbeva P, van Veen JA, van Elsas JD (2003) Predominant Bacillus spp. in Agricultural Soil under Different Management Regimes Detected via PCR-DGGE. Microb Ecol 45(3):302-316

Goto K, Omura T, Hara Y, Sadaie Y (2000) Application of the partial 16S rDNA sequence as an index for rapid identification of the species in the genus Bacillus. J Gen Appl Microbiol 46:1-8

Gurtler V, Stanisich VA (1996) New approaches for typing and identification of bacteria using the 16S-23S rDNA spacer region. Microbiology 142:3-16

Heuer H, Smalla K (1997) Application of denaturing gradient gel electrophoresis (DGGE) and temperature gradient gel electrophoresis (TGGE) for studying soil microbial com-munities. In: Van Elsas JD, Trevors JT, Wellington EMH (eds) Modern Soil Microbiology. Marcel Dekker, New York, pp 353-373

Heyndrickx M, Lebbe L, Kersters K, Devos P, Forsyth G (1998) Virgibacillus: a new genus to accommodate Bacillus pantothenticus (Proom and Knight 1950). Emended description of Virgibacillus pantothenticus. Int J Syst Bacteriol 48:99-106

Kadyan S, Panghal M, Kumar S, Singh K, Yadav JP (2013) Assessment of functional and genetic diversity of aerobic endospore forming Bacilli from rhizospheric soil of Phyllanthus amarus L. World J Microbiol Biotechnol, doi:10.1007/ s11274-013-1323-3

Kumar P, Khare S, Dubey RC (2012) Diversity of Bacilli from Disease Suppressive Soil and their Role in Plant Growth Promotion and Yield Enhancement. New York Sci J 5(1):90-111

Larkin MA, Blackshields G, Brown NP, Chenna R, McGettigan PA et al (2007) Clustal W and Clustal X version 2.0. Bioinformatics 23:2947-2948

Mandic-Mulec I, Prosser JI (2011) Diversity of Endospore-forming Bacteria in Soil: Characterization and Driving Mechanisms. In: Logan NA, De Vos P (eds) Endospore-forming Soil Bacteria, Soil Biology 27. Springer-Verlag, Berlin Heidelberg, pp 31-59

Nazina TN, Tourova TP, Poltaraus AB, Novikova EV, Grigoryan AA et al (2001) Taxonomic study of aerobic thermophilic bacilli: descriptions of Geobacillus subterraneus gen. nov. sp. nov. and Geobacillus uzenensis sp. nov. from petroleum reservoirs and transfer of Bacillus stearothermophilus, Bacillus thermocatenulatus, Bacillus thermoleovorans, Bacillus kaustophilus, Bacillus thermoglucosidasius, Bacillus thermodenitrificans to Geobacillus as Geobacillus stearothermophilus, Geobacillus thermocatenulatus, Geobacillus thermoleovorans, Geobacillus kaustophilu, Geobacillus thermoglucosidasius, Geobacillus thermodenitrificans. Int J Syst Evol Microbiol 51:433-446

Niimura Y, Koh E, Yanagida F, Suzuki Kl, Komagata K et al (1990) Amphibacillus xylanus gen. nov., a facultatively anaerobic spore foming xylin-digesting bacterium which lacks cytochrome, quinone, and catalase. Int J Syst Bacteriol 40:297-301

Priest FG, Goodfellow M, Todd C (1988) A numerical classification of the genus Bacillus. J Gen Microbiol 134:1847-1882

Schlesner H, Lawson PA, Collins MD, Weiss N, Wehmeyer U et al (2001) Filobacillus milensis gen. nov., a new halophilic spore-forming bacterium with Ornd- Glu-type peptidoglycan. Int J Syst Microbiol 51:425-431

Shida O, Takagi H, Kadowaki K, Yano H, Komagata K (1996) Proposal for two new genera, Brevibacillus gen. Nov. and Aneurinibacillus gen. nov. Int J Syst Bacteriol 46:939-946

Stackebrandt E, Swiderski J (2002) From phylogeny to systematics: The dissection of the genus Bacillus. In: Berkeley R, Heyndrickx M, Logan N, De Vos P (eds) Applications and systematics of Bacillus and relatives. Blackwell, Malden, p 822

Tamura K, Peterson D, Peterson N, Stecher G, Nei M, Kumar S (2011) MEGA5: Molecular Evolutionary Genetics Analysis Using Maximum Likelihood, Evolutionary Distance and Maximum Parsimony Method. Mol Biol Evol 28:2731-2739

Van Elsas JD, Garbeva P, Salles J (2002) Effects of agricultural measures on the microbial diversity of soil as related to suppression of soil-borne plant pathogens. Biodegradation 13:29-40

Vardhan S, Kaushik R, Saxena AK, Arora DK (2011) Restriction analysis and partial sequencing of the $16 \mathrm{~S}$ rRNA gene as index for rapid identification of Bacillus species. Antonie Van Leeuwenhoek 99:283-296

Waino M, Tindall BJ, Schumann P, Ingvorsen K (1999) Gracillbacillus gen. nov., with description of Graclibacillus holotolerence gen. nov., and Bacillus salexigens to the genus Salibacillus gen. nov., as Salibacillus salexigens comb. Nov. Int J syst bacteriol 49:821-831
Wisotzkey JD, Jurtshuk P Jr, Fox GE, Deinhard G, Poralla K (1992) Comparative sequence analyses on the 16S r RNA (rDNA) of Bacillus acidocaldarius, Bacillus acidoterrestris, and Bacillus cycloheptanicus and proposal for certain of a new genus, Alicyclobacillus gen. nov. Int J Syst Bacteriol 42:263-269

Xu D, Cote JC (2003) Phylogenetic relationships between Bacillus species and related genera inferred from comparison of $3^{\prime}$ end $16 \mathrm{~S}$ rDNA and $5^{\prime}$ end 16S-23S ITS nucleotide sequences. Int J Syst Evol Microbiol 53:695-704

Yarza PW, Ludwig J, Euzeby R, Amann KH, Schleifer FO et al (2010) Update of the All-Species Living Tree Project based on 165 and 235 rRNA sequence analyses. Syst Appl Microbiol 33:291-299

Yoon JH, Weiss N, Lee KC, Lee IS, Kang KH et al (2001) Jeotgalibacillus alimentarius gen. nov., sp. Nov., a novel bacterium isolated from jeotgal with L-lysine in the cell wall, reclassification of Bacillus marinus (Ruger 1983) as Marinibacillus marinus gen. nov., comb. Nov. Int J Syst Evol Microbiol 51:2087-2093

doi:10.1186/2193-1801-2-596

Cite this article as: Kadyan et al:: Development of a PCR based marker system for easy identification and classification of aerobic endospore forming bacilli. SpringerPlus 2013 2:596.

\section{Submit your manuscript to a SpringerOpen ${ }^{\circ}$ journal and benefit from:}

- Convenient online submission

Rigorous peer review

- Immediate publication on acceptance

- Open access: articles freely available online

- High visibility within the field

- Retaining the copyright to your article

Submit your next manuscript at $>$ springeropen.com 